Supporting Information

\title{
Mechanisms of Reaction of Aminoxyl (Nitroxide), Iminoxyl and Imidoxyl Radicals with Alkenes and Proof that in the Presence of Lead Tetraacetate, N- Hydroxyphthalimide Reacts with Alkenes by Both Radical and Non-Radical Mechanisms
}

Sergiu Coseri, ${ }^{\star \dagger}$ G. David Mendenhall ${ }^{\ddagger}$ and K. U. Ingold

National Research Council, Ottawa, ON, Canada K1A 0R6

${ }^{\dagger}$ Permanent address: P. Poni Institute of Macromolecular Chemistry, Gr. Ghica Voda Alley, 41A, 6600, Iasi, Roumania; E-mail: coseris@icmpp.ro

‡Eastern Sources, Inc., 8 Westchester Plaza, Elmsford, NY 10523, USA

\section{Contents}

\begin{tabular}{|c|c|}
\hline Page(s) & Item \\
\hline S2 & $\begin{array}{l}\text { Experimental procedures for the syntheses of 1,2-dideuterio-cyclooctene and } \\
\text { trans-3,4-dideuteriohex-3-ene. }\end{array}$ \\
\hline S3-S4 & LFP and competition kinetic results. \\
\hline S5-S8 & $\begin{array}{l}{ }^{1} \mathrm{H} \text { NMR and }{ }^{13} \mathrm{C} \text { NMR spectra in } \mathrm{CDCl}_{3} \text { of the cyclooctene / PINO mono- and } \\
\text { di-adducts. }\end{array}$ \\
\hline $\begin{array}{l}\text { S21-S22 } \\
\text { S23-S28 }\end{array}$ & $\begin{array}{l}{ }^{2} \mathrm{H} \mathrm{NMR} \text { spectra in } \mathrm{CHCl}_{3} \text { of the products of the following reactions: di-tert- } \\
\text { butyliminoxyl }+1,2 \text {-dideuteriocyclohexene, PINO }+1,2 \text {-dideuteriocyclohexene, } \\
\text { PINO }+1,2 \text {-dideuteriocyclooctene, (neat trans-3,4-dideuteriohex-3-ene), } 4- \\
\text { hydroxyTempo }+ \text { trans-3,4-dideuteriohex-3-ene, di-tert-butyliminoxyl }+ \text { trans- } \\
3,4 \text {-dideuteriohex-3-ene, PINO }+ \text { trans-3,4-dideuteriohex-3-ene and of an } \\
\text { equimolecular mixture of the trans-3,4-dideuteriohex-3-ene and trans-3,4- } \\
\text { dideuteriohex-3-ene-PINO di-adduct. } \\
\text { Comparison of the Cl-MS M }+1 \text { peaks for the cyclooctene reaction with NHPI in } \\
\text { the presence of lead tetraacetate, using } 2 \text { different protocols (see page 18). } \\
\text { X-ray crystallographic data for cyclooctene / PINO mono-adduct. }\end{array}$ \\
\hline S29-S33 & X-ray crystallographic data for cyclooctene / PINO di-adduct. \\
\hline
\end{tabular}




\section{Syntheses of dideuterioalkenes}

1,2-Dideuteriocyclooctene was synthesized by reduction of cyclooctyne (prepared from cyclooctene, according to the literature ${ }^{1}$ ) using lithium aluminum deuteride. In a 200 $\mathrm{mL}$ round bottom flask fitted with a reflux condenser, a slurry of $\mathrm{LiAID}_{4}(2.52 \mathrm{~g}, 60 \mathrm{mmol})$ in $60 \mathrm{~mL}$ anhydrous ether was prepared. To the slurry, was added cyclooctyne $(5.41 \mathrm{~g}$, $50 \mathrm{mmol}$ ) dropwise via syringe. The heterogeneous mixture was refluxed for 8 hours. The mixture was allowed to cool, the flask was placed in an ice bath, and then carefully quenched by dropwise addition of $\mathrm{D}_{2} \mathrm{O}(12 \mathrm{~mL}), \mathrm{D}_{2} \mathrm{O} / \mathrm{DCl}(20 \% \mathrm{DCl}, 8 \mathrm{~mL})$ and finally $\mathrm{HCl}(6 \mathrm{~N}, 20 \mathrm{~mL})$. The organic layer was washed with water $(5 \times 12 \mathrm{~mL})$, dried with $\mathrm{Na}_{2} \mathrm{SO}_{4}$ and filtered. The solvent was removed at $0{ }^{\circ} \mathrm{C}$, using a water pump. The residue was distilled under reduced pressure to give $3.37 \mathrm{~g} \mathrm{(30} \mathrm{mmol,} 60 \%$ yield) of the title compound as a clear colorless liquid.

${ }^{1} \mathrm{H}$ NMR $\left(\mathrm{CDCl}_{3}\right): \delta 2.16(8 \mathrm{H}), 1.52(4 \mathrm{H}) ;{ }^{13} \mathrm{C} \mathrm{NMR}\left(\mathrm{CDCl}_{3}\right): \delta 130.3(\mathrm{t}), 77.4,26.5$

Deuterium incorporation at the vinylic site was $>99 \%$ as determined by ${ }^{1} \mathrm{H}$ NMR.

Trans-3,4-dideuteriohex-3-ene was prepared following a modified literature method. ${ }^{2}$

In a round-bottom flask fitted with a reflux condenser a slurry of $\mathrm{LiAID}_{4}(1.4 \mathrm{~g}, 33.6$ $\mathrm{mmol}$ ) in $30 \mathrm{~mL}$ anhydrous diglyme was prepared. To the slurry was added 3-hexyne $(2.4 \mathrm{~g}, 28.6 \mathrm{mmol})$ dropwise via syringe. The heterogeneous mixture was heated to $150{ }^{\circ} \mathrm{C}$ for 6.5 hours. The mixture was allowed to cool to room temperature, the flask was placed in an ice-bath, and then carefully quenched by dropwise addition of $\mathrm{D}_{2} \mathrm{O}(7$ $\mathrm{mL}), \mathrm{D}_{2} \mathrm{O} / \mathrm{DCl}(20 \% \mathrm{DCl}, 3 \mathrm{~mL})$ and finally $\mathrm{HCl}(6 \mathrm{~N}, 10 \mathrm{~mL})$. The dideuterio compound was extracted into hexadecane, and the hexadecane layer was washed with water ( $5 \mathrm{x}$ $8 \mathrm{~mL}$ ), dried with $\mathrm{Na}_{2} \mathrm{SO}_{4}$ and filtered. Distillation gave $1.24 \mathrm{~g}$ (14.4 mmol, $50 \%$ yield) of the title compound as a clear, colorless liquid.

${ }^{1} \mathrm{H}$ NMR $\left(\mathrm{CHCl}_{3}\right) \delta 1.99\left(\mathrm{q}, 4 \mathrm{H}, \mathrm{CH}_{2}\right), 1.00\left(\mathrm{t}, 6 \mathrm{H}, \mathrm{CH}_{3}\right) ;{ }^{13} \mathrm{C} \mathrm{NMR}\left(\mathrm{CHCl}_{3}\right) \delta 130.4(\mathrm{t})$, 25.4, 13.9 .

Deuterium incorporation at the vinylic site was $>99 \%$ as determined by ${ }^{1} \mathrm{HNMR}$.

\footnotetext{
${ }^{1}$ Brandsma, L.; Verkruijsse, H. D. Synthesis 1978, 290

${ }^{2}$ Kroll, J. H.; Donahue, N. M.; Cee, V. J.; Demerjian, K. L.; Anderson, J. G. J. Am. Chem. Soc. 2002, 124, 85188519
} 
Cumyl alcohol $(\mathbf{A})$ / acetophenone $(\mathbf{K})$ ratio upon thermal decomposition of dicumyl hyponitrite $(0.015 \mathrm{mmol})$ in the presence of different concentration of NHPI in benzene, at $30{ }^{\circ} \mathrm{C}$

$\begin{array}{cc}10^{3} \times[\mathbf{N H P I}] / \mathrm{M} & \mathbf{A} / \mathbf{K} \\ 0.15 & 0.169 \\ 0.45 & 0.475 \\ 0.75 & 1.169 \\ 1.05 & 2.762 \\ 1.35 & 3.166\end{array}$

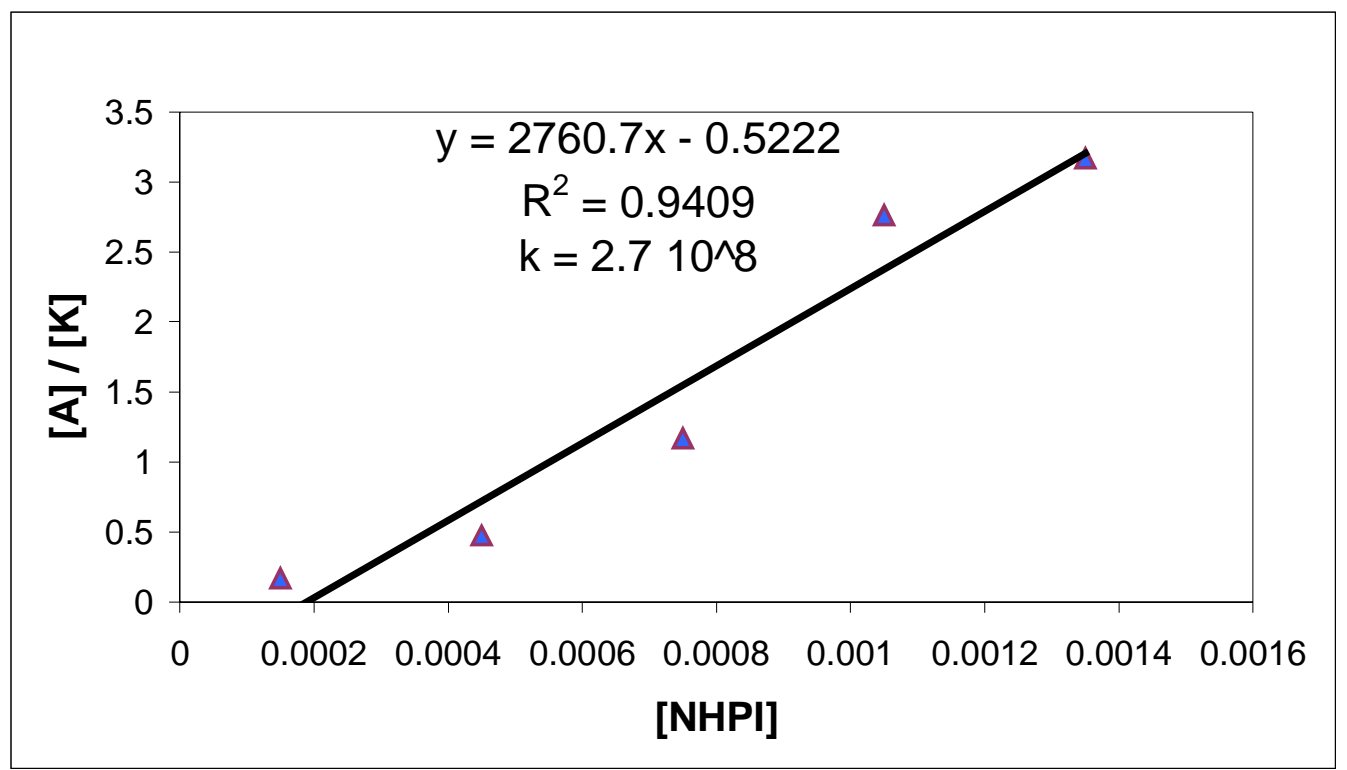

Figure S1. Plot of GC/FID determined ratios of cumyl alcohol (A) / acetophenone (K) versus NHPI concentration 
LFP data for reaction of the cumyloxyl radical with three non-deuterated alkenes at 25 ${ }^{\circ} \mathrm{C}$ in benzene. Numbers in bold face are $k / \mathrm{M}^{-1} \mathrm{~s}^{-1}$, the calculated bimolecular rate constant for each data set with $\mathrm{R}^{2}$ in parenthesis

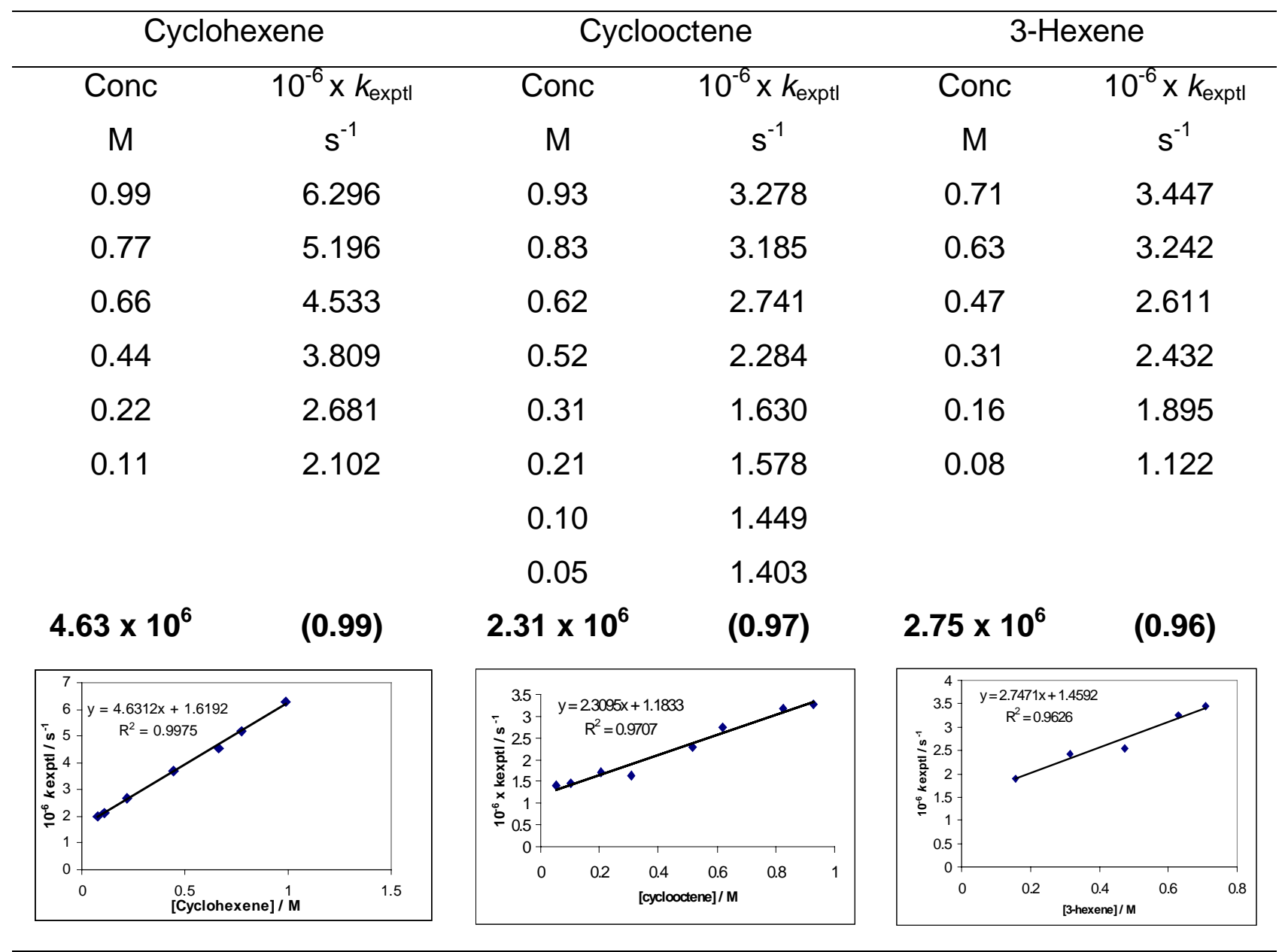




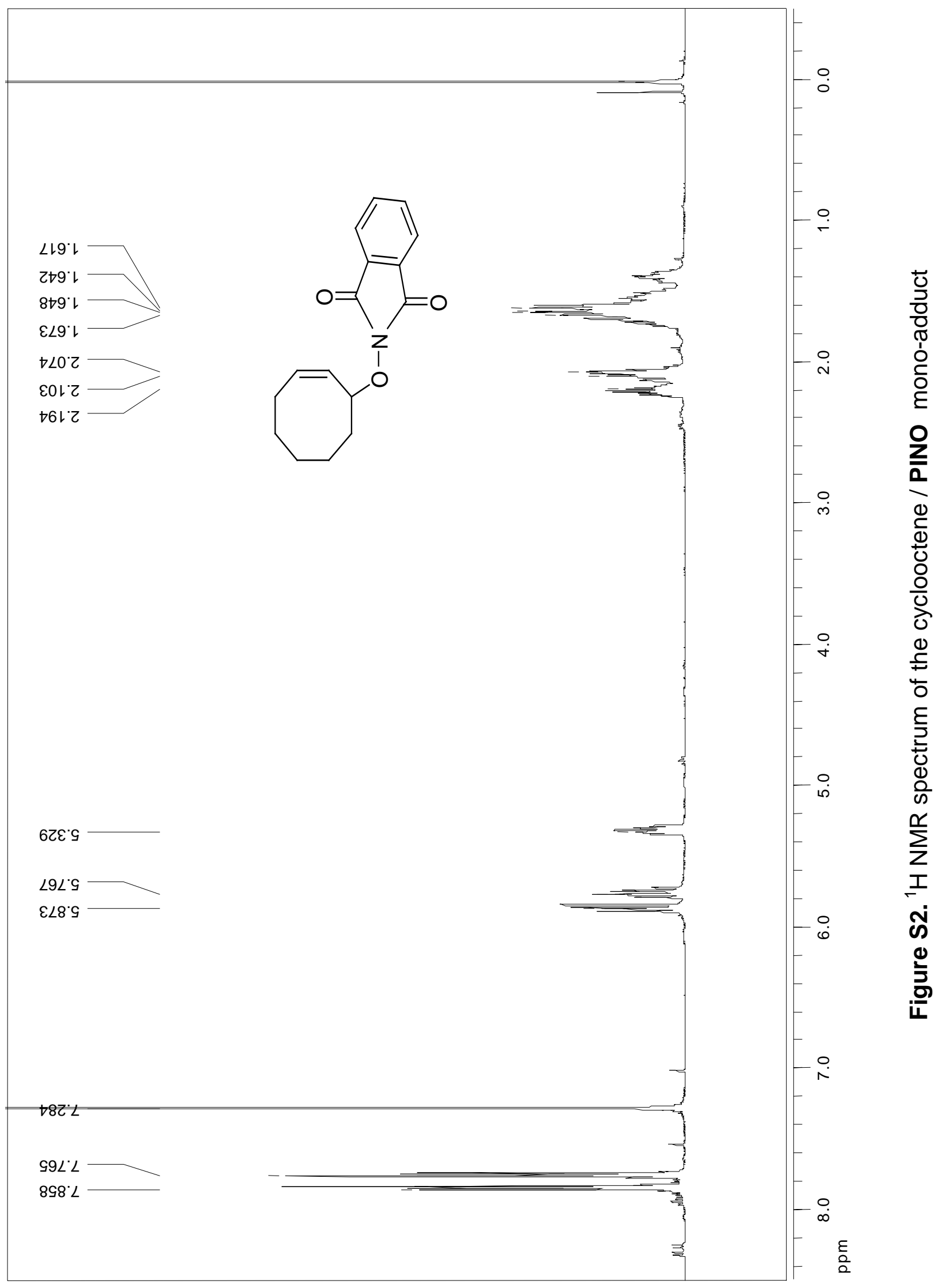




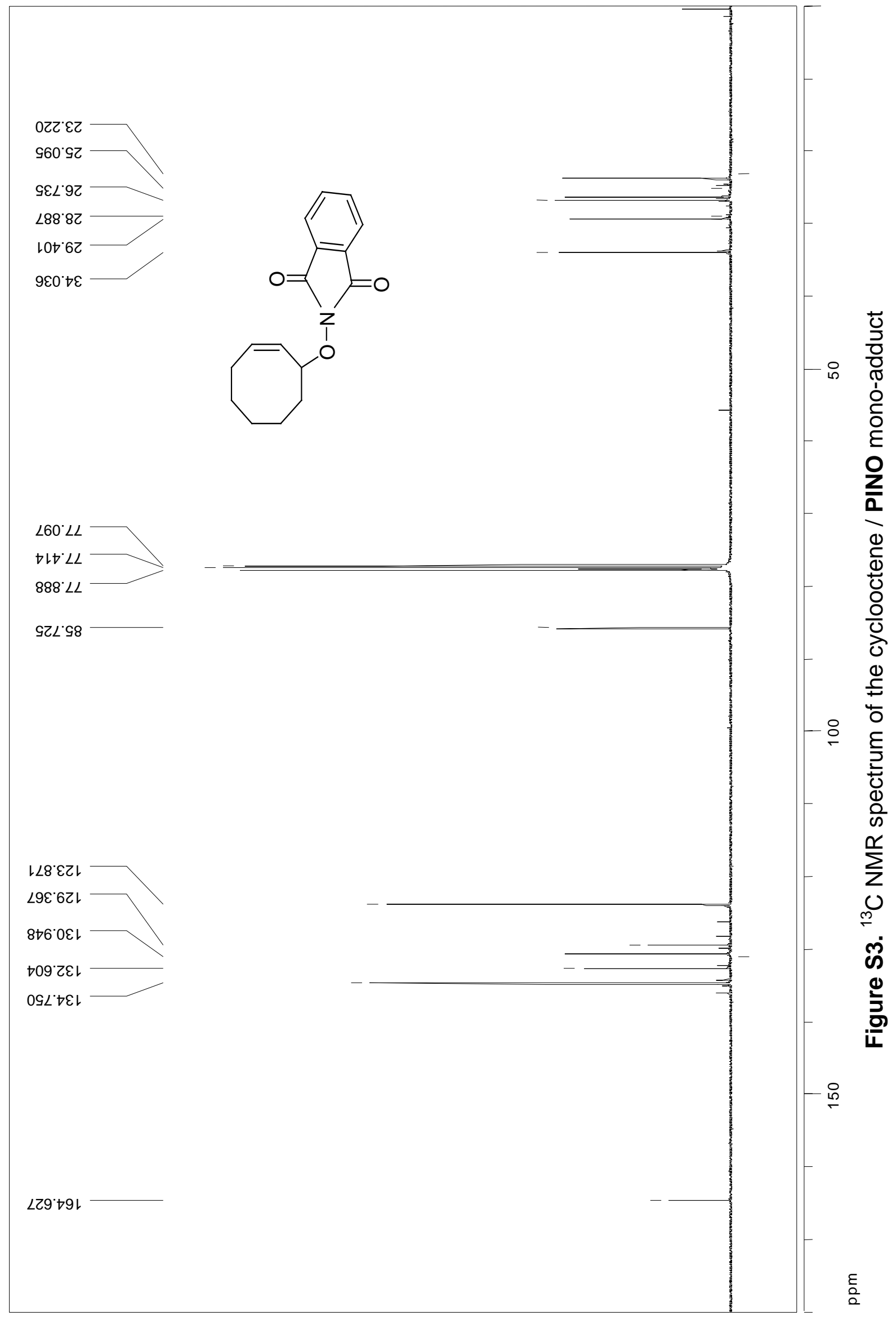




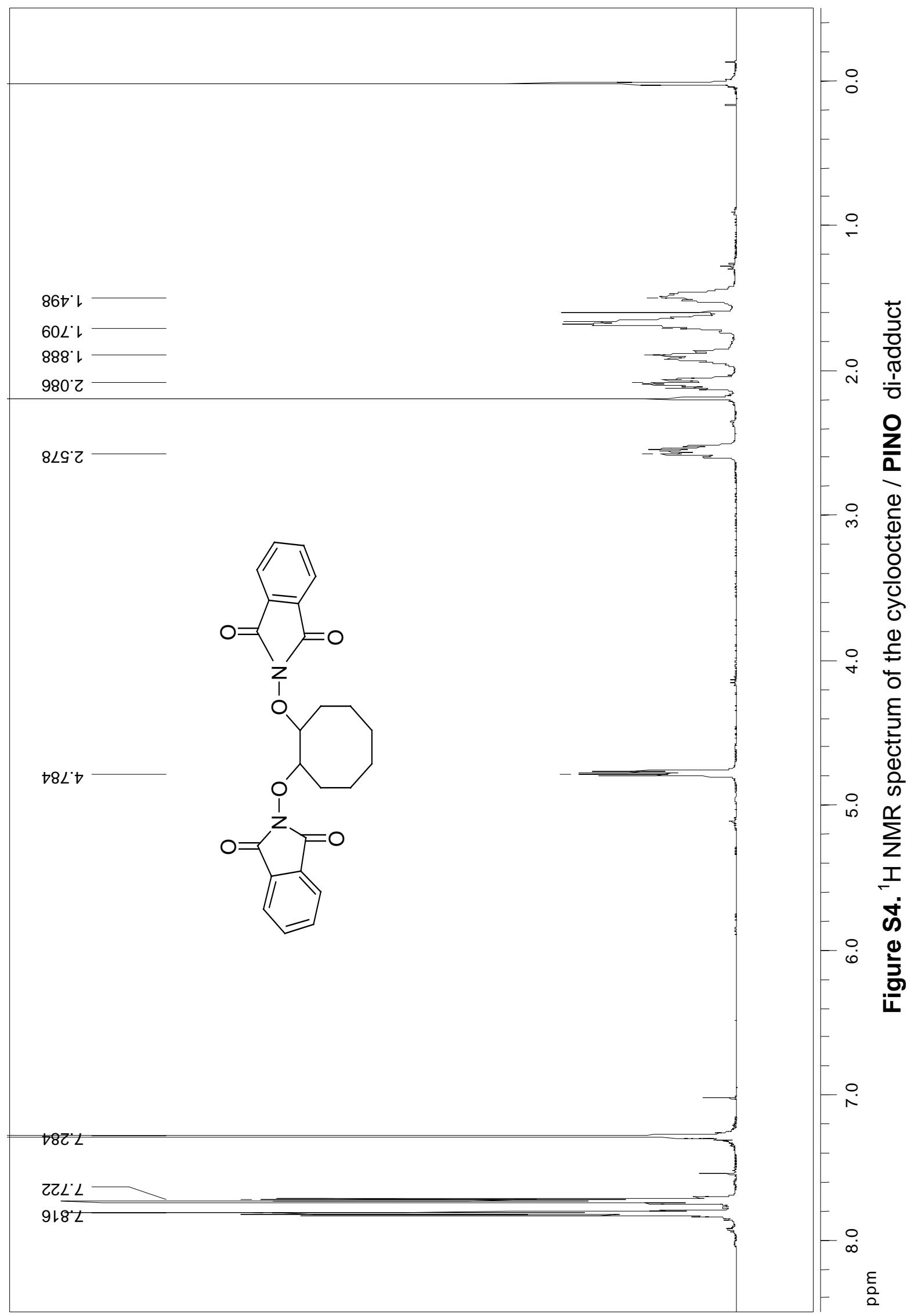




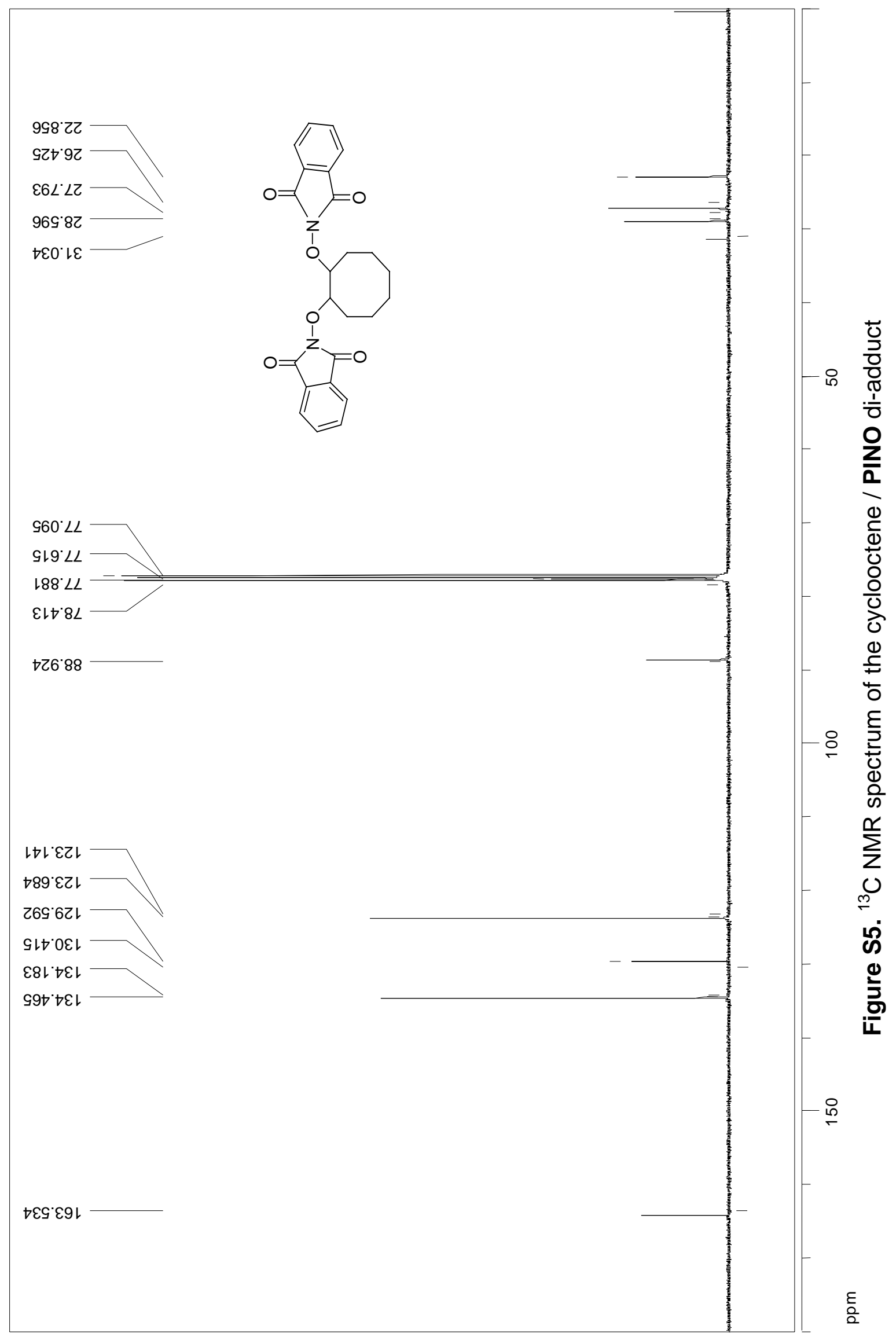




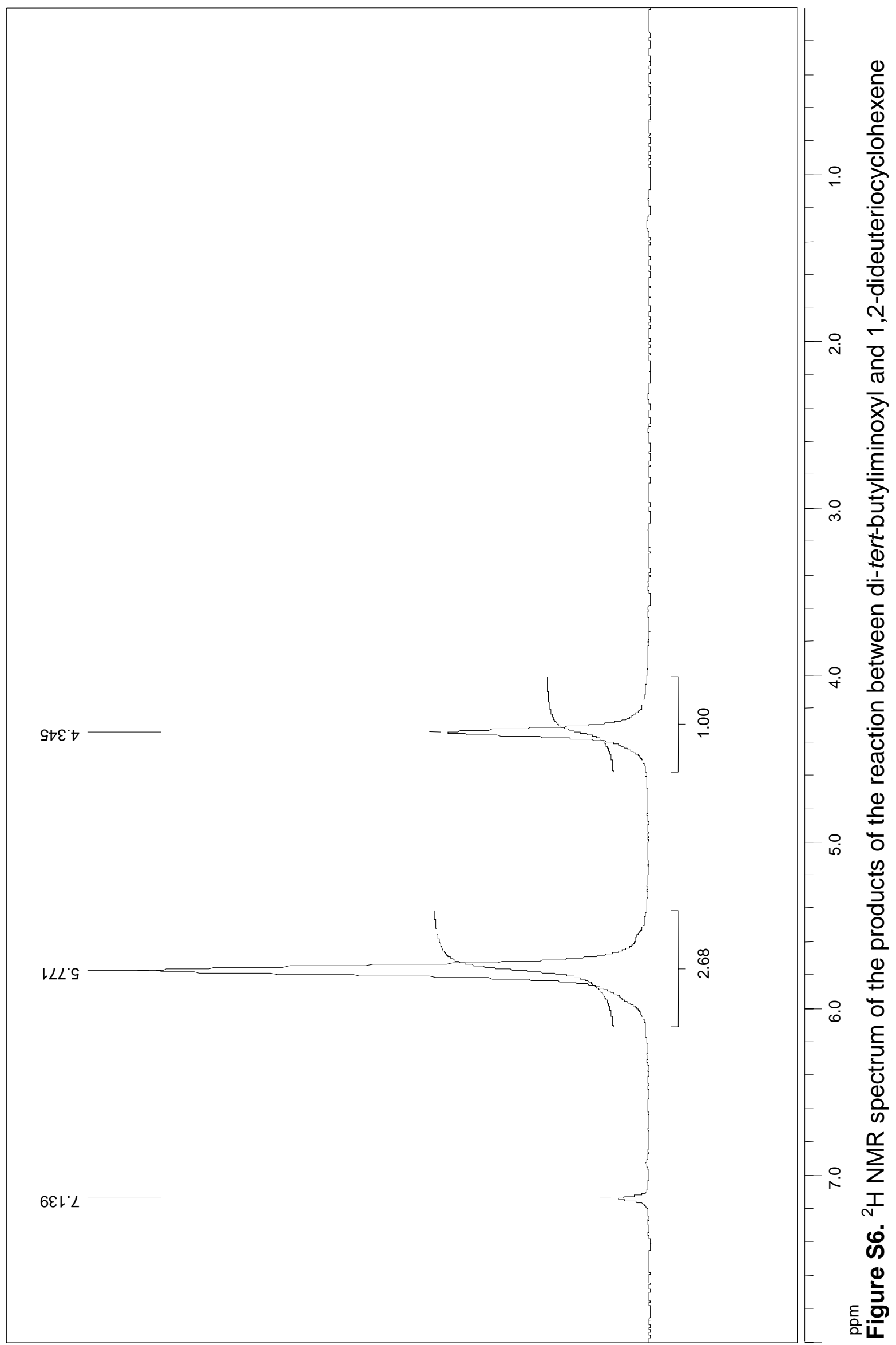




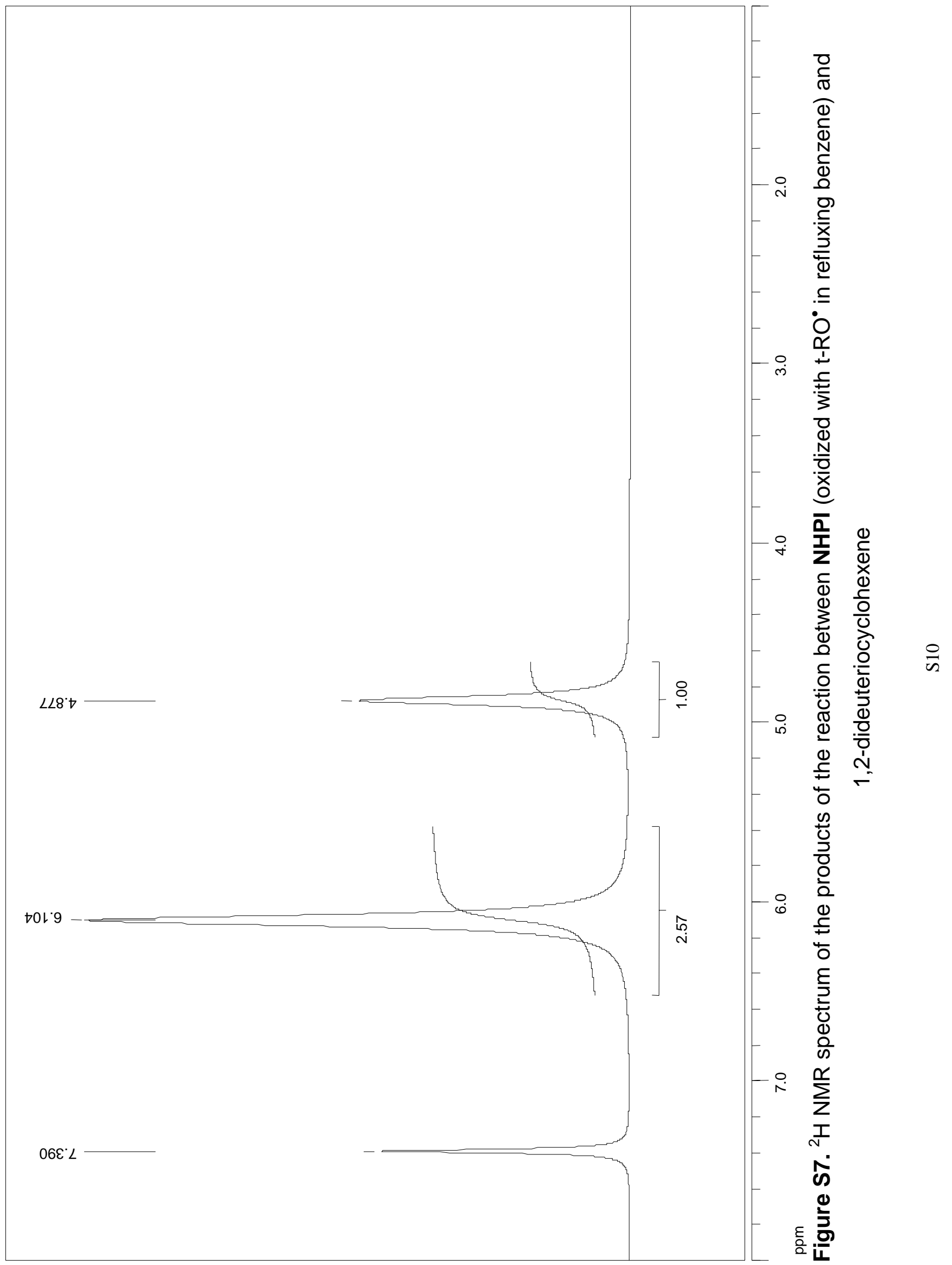




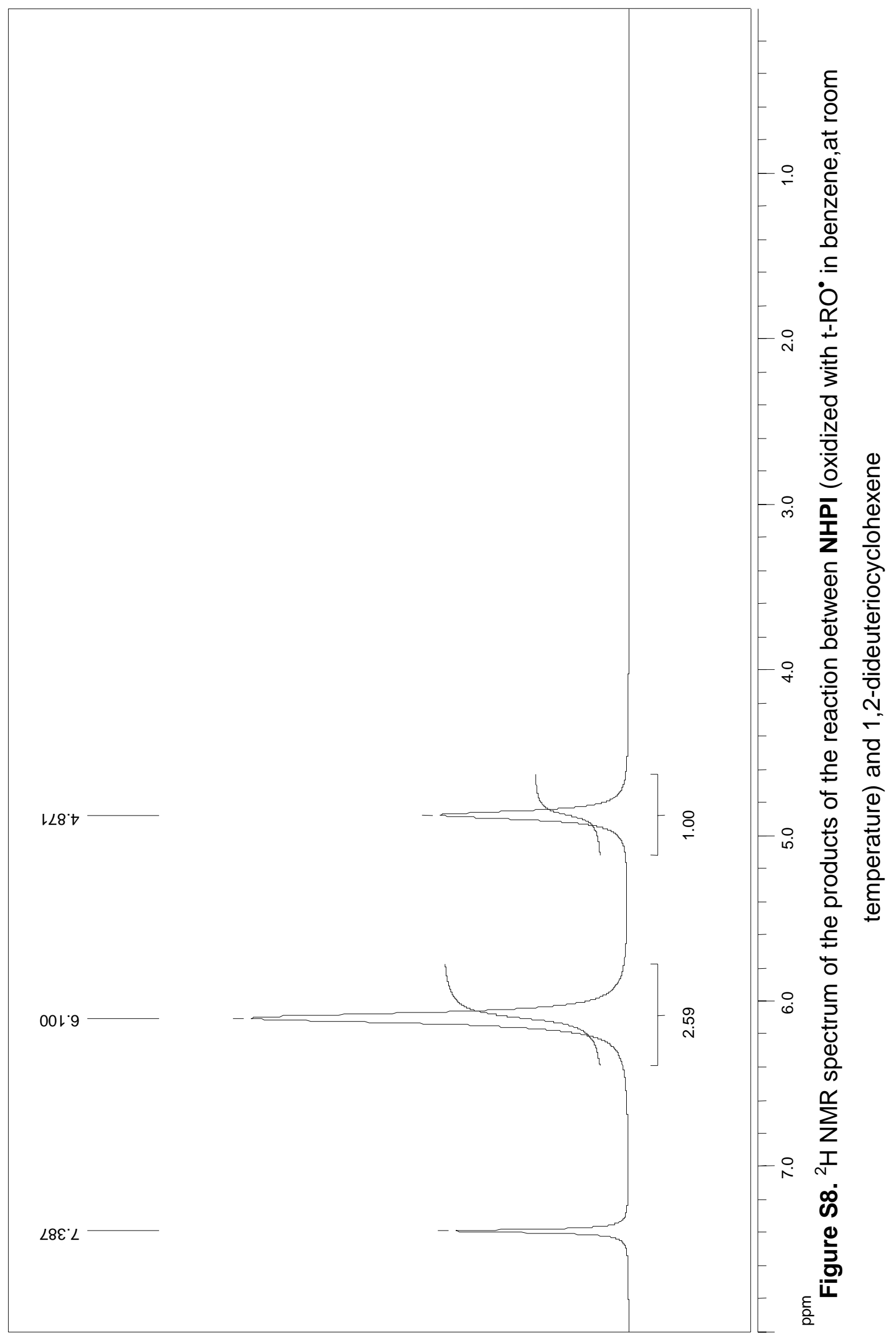




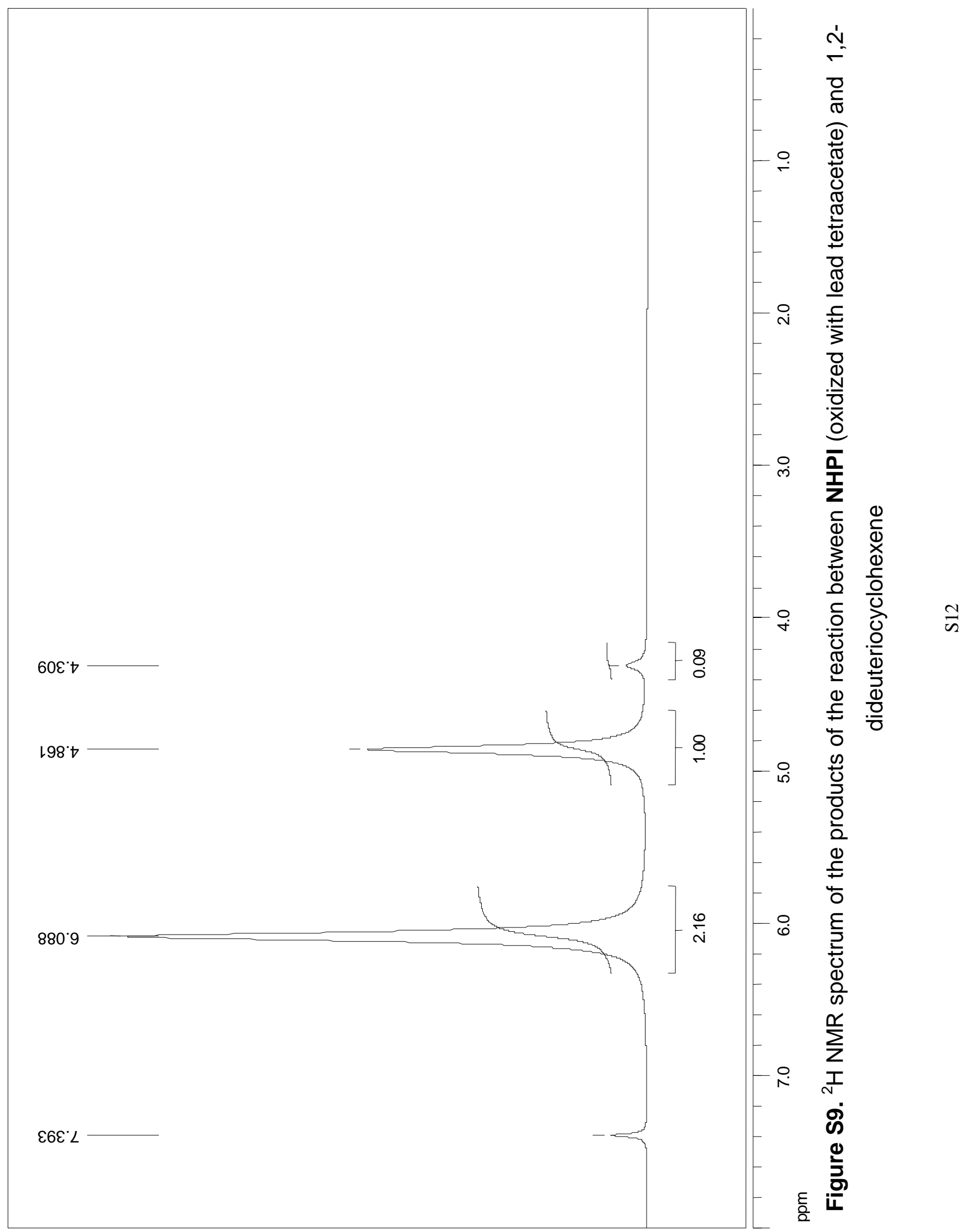




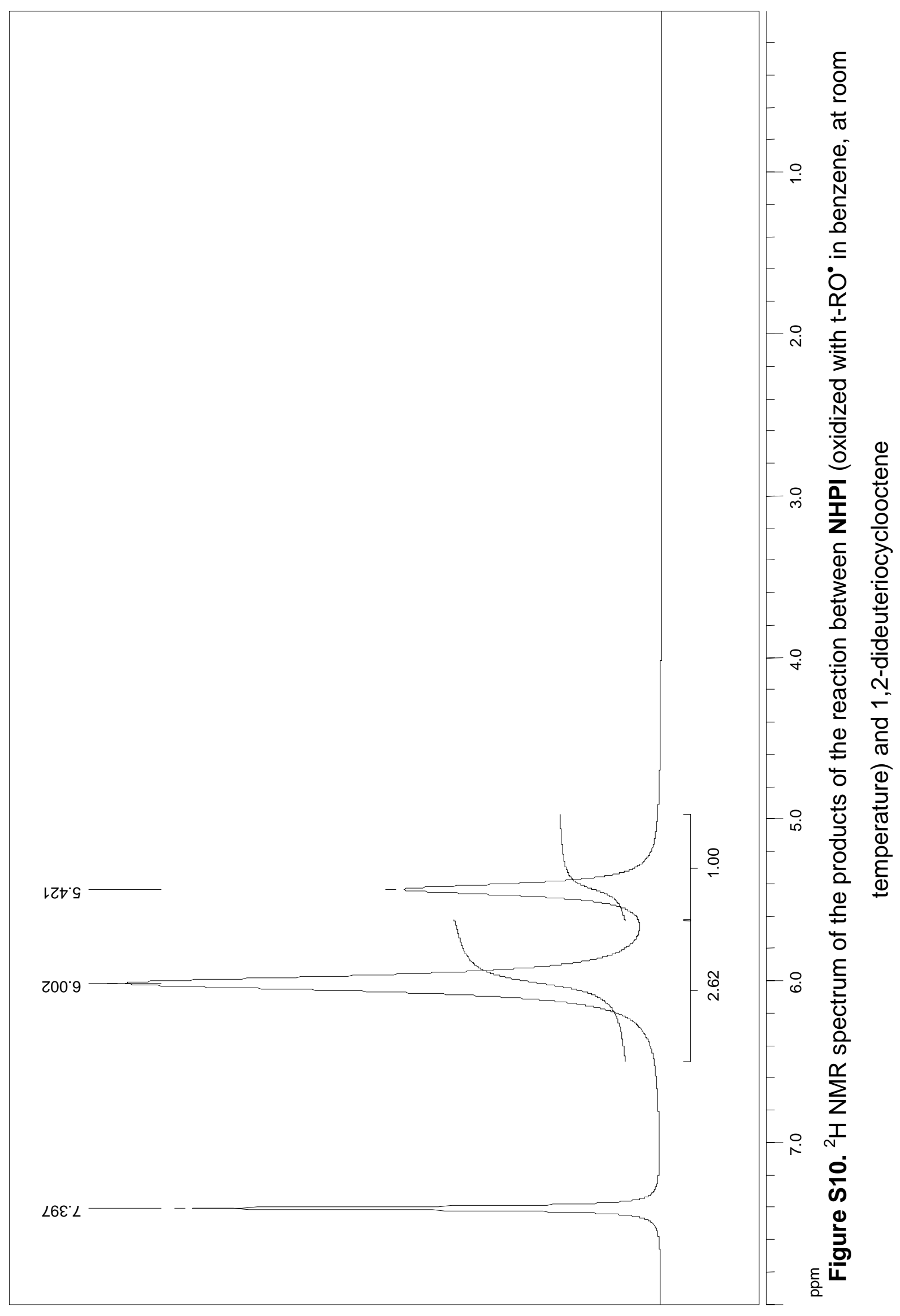




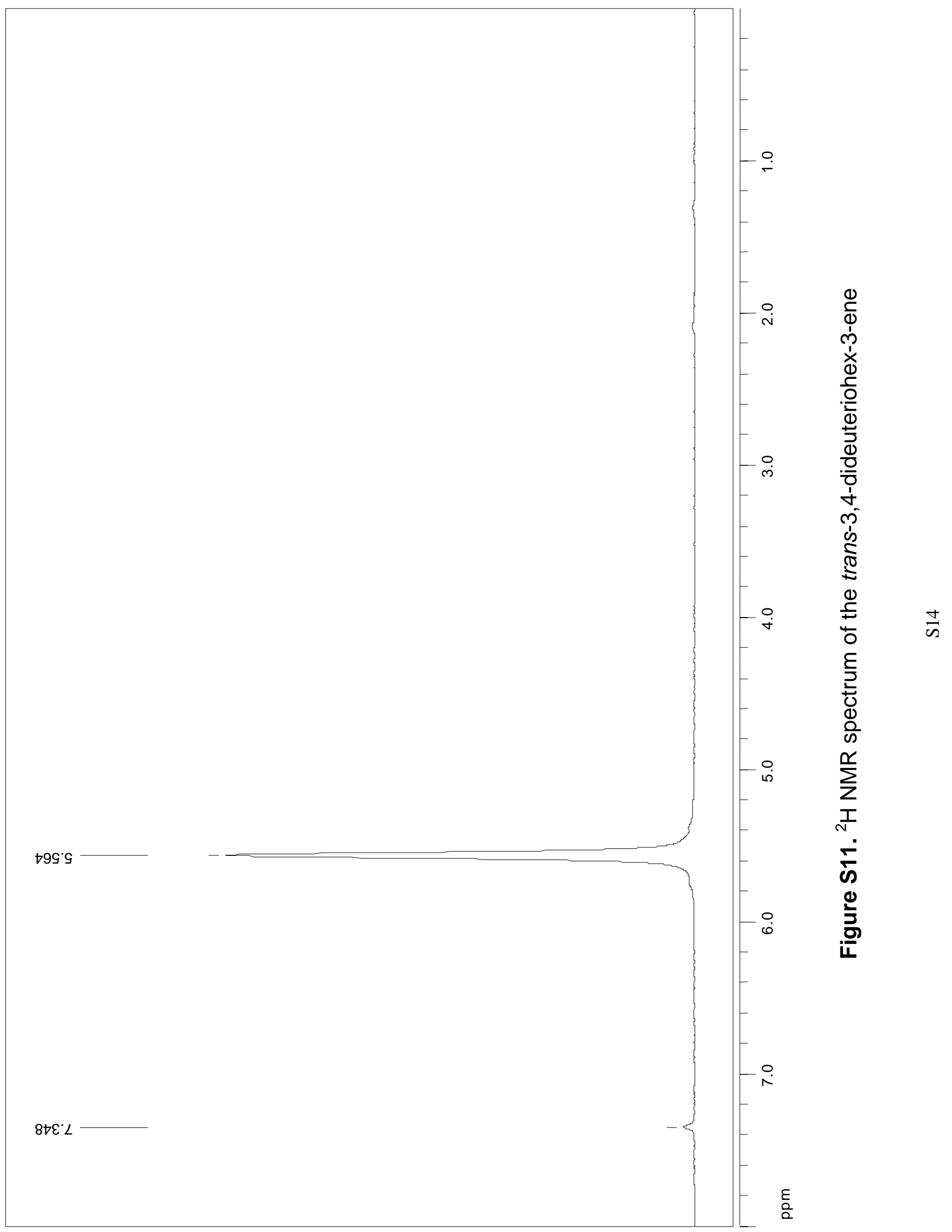




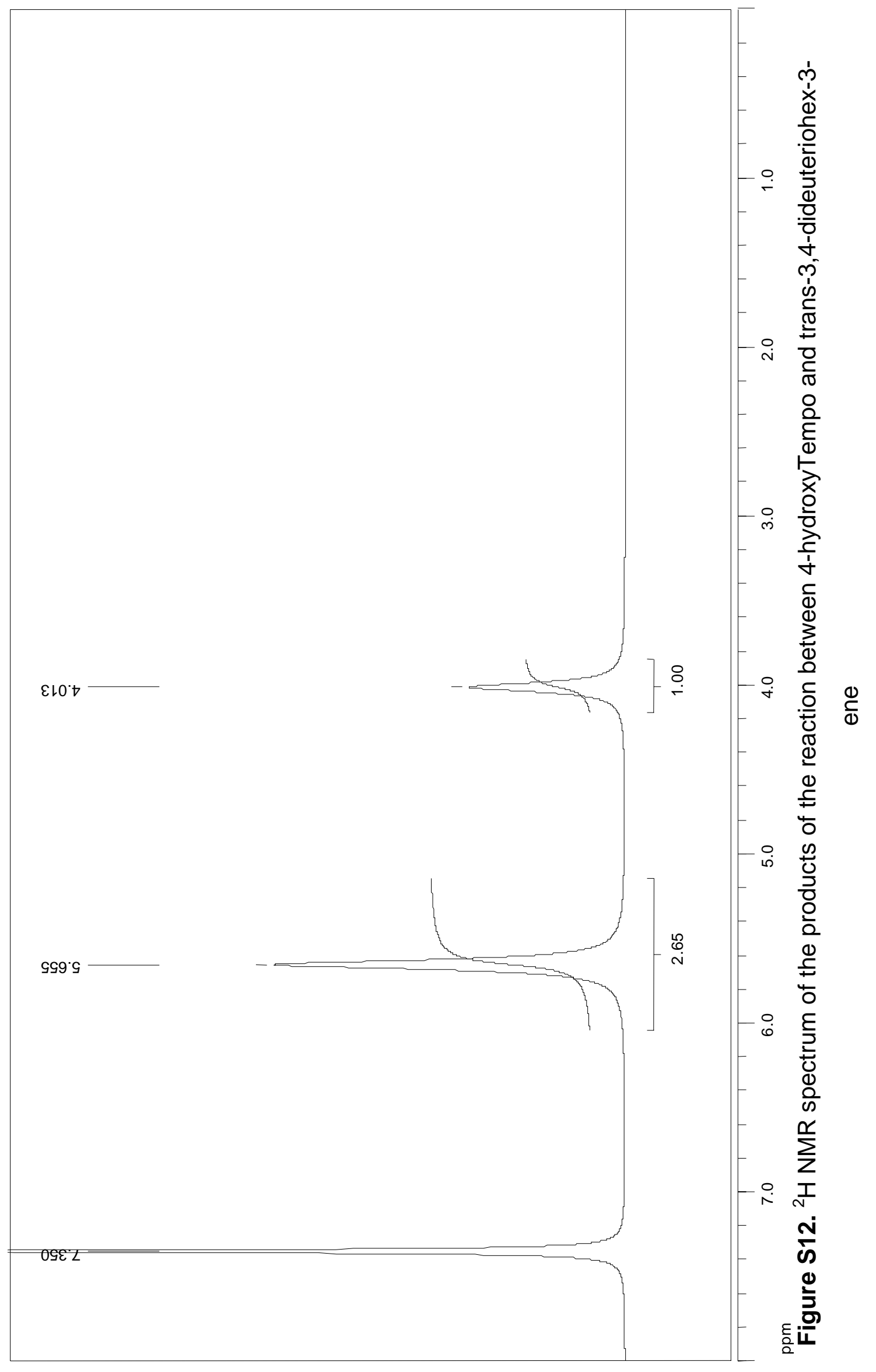




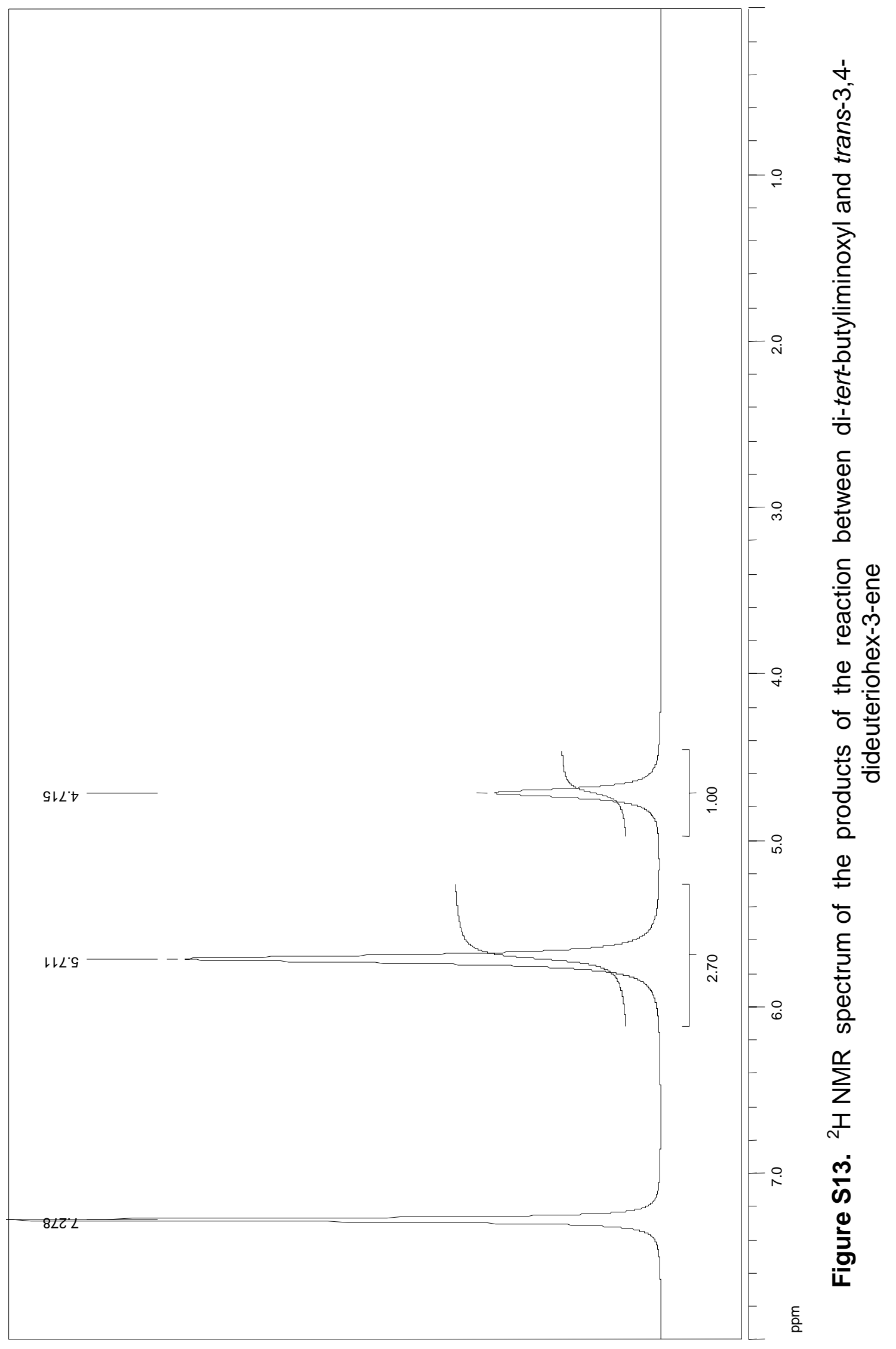




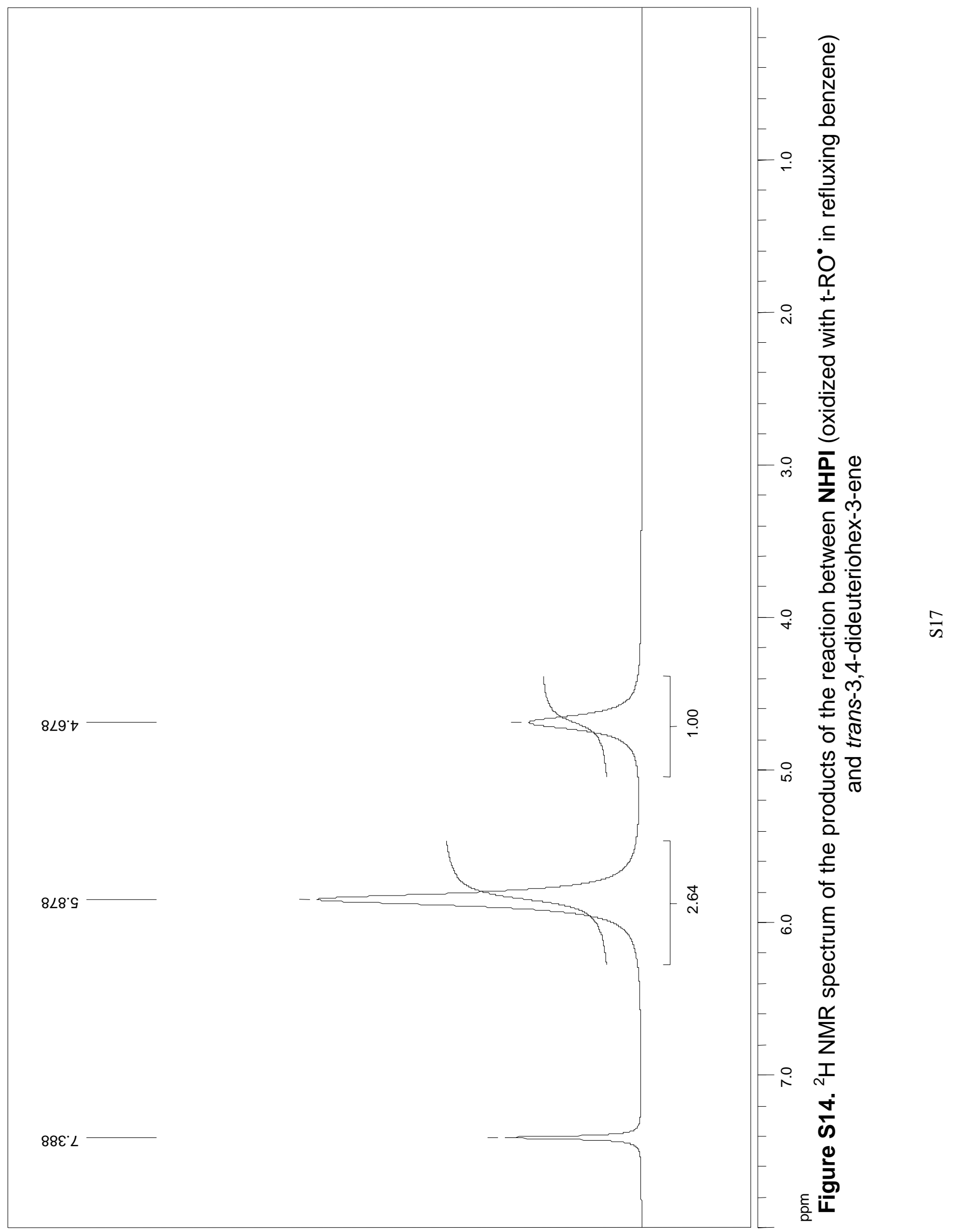




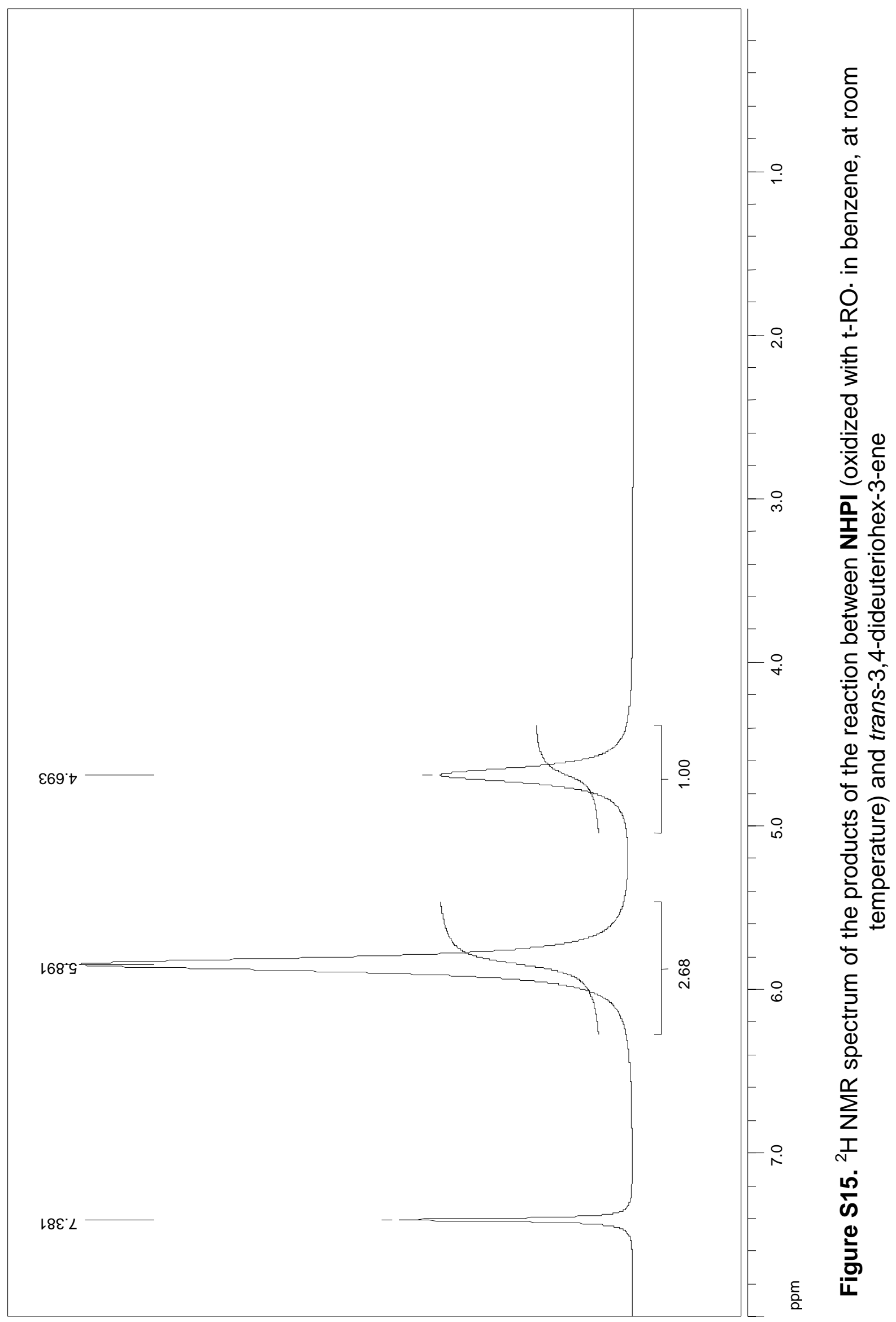




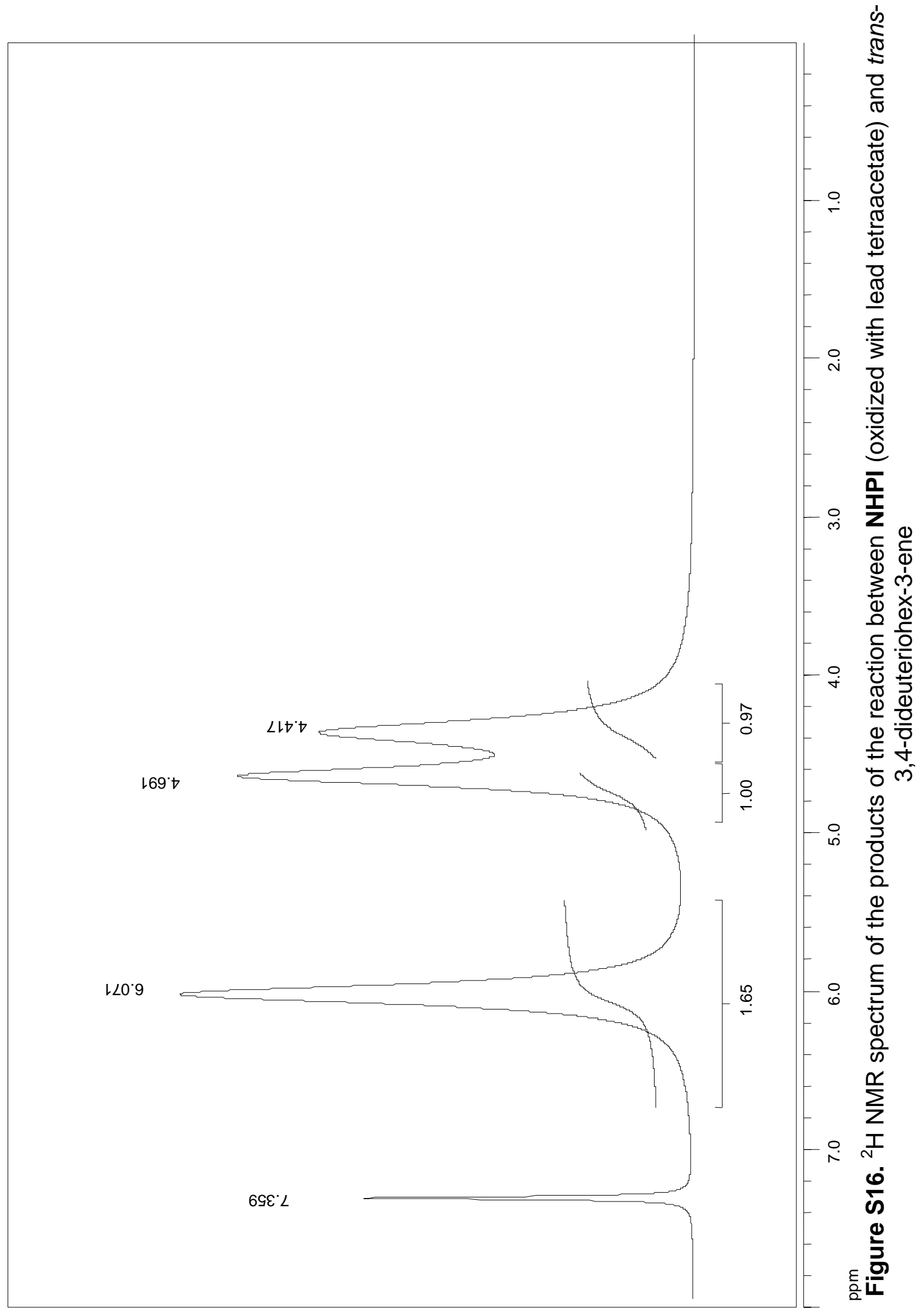




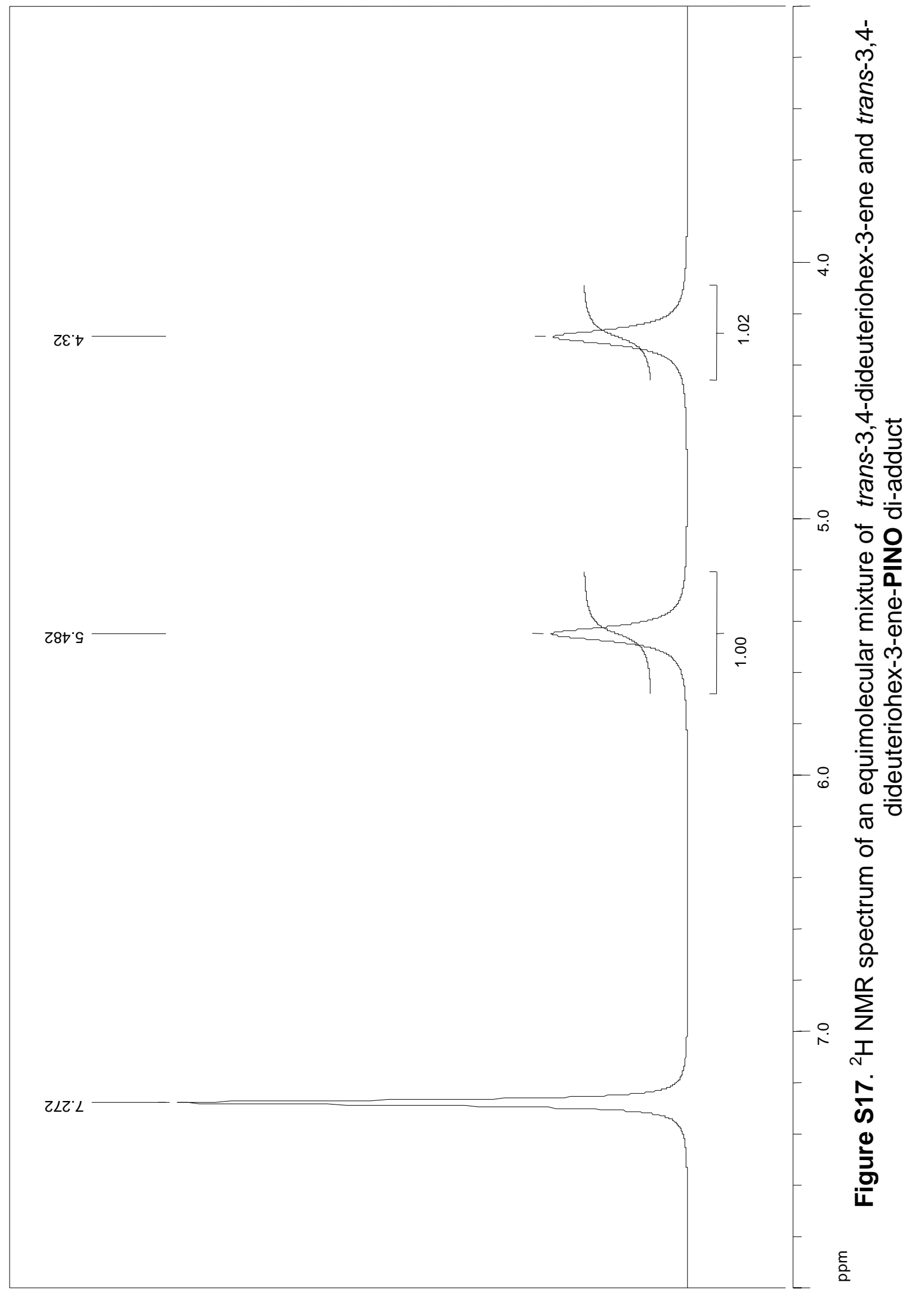



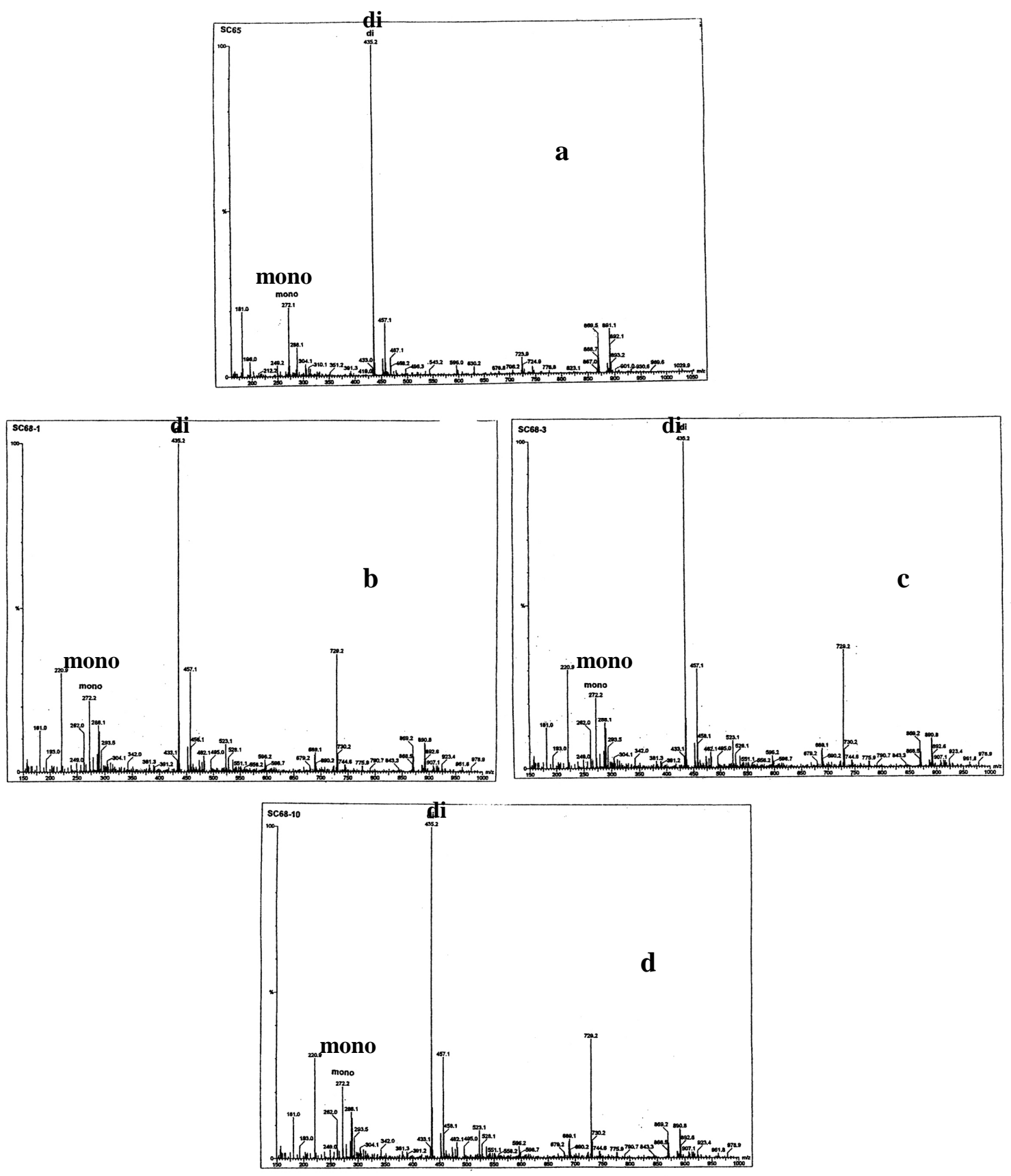

Figure S18. Comparison of the CI-MS $\mathrm{M}+1$ peaks for the cyclooctene reaction with NHPI in the presence of lead tetraacetate a) original experiment, b) protocol 1,1 minute prior the addition of cyclooctene (see page 18), c) protocol 1, 3 minutes prior the addition of cyclooctene (see page 18), d) protocol 1, 10 minutes prior the addition of cyclooctene (see page 18) 

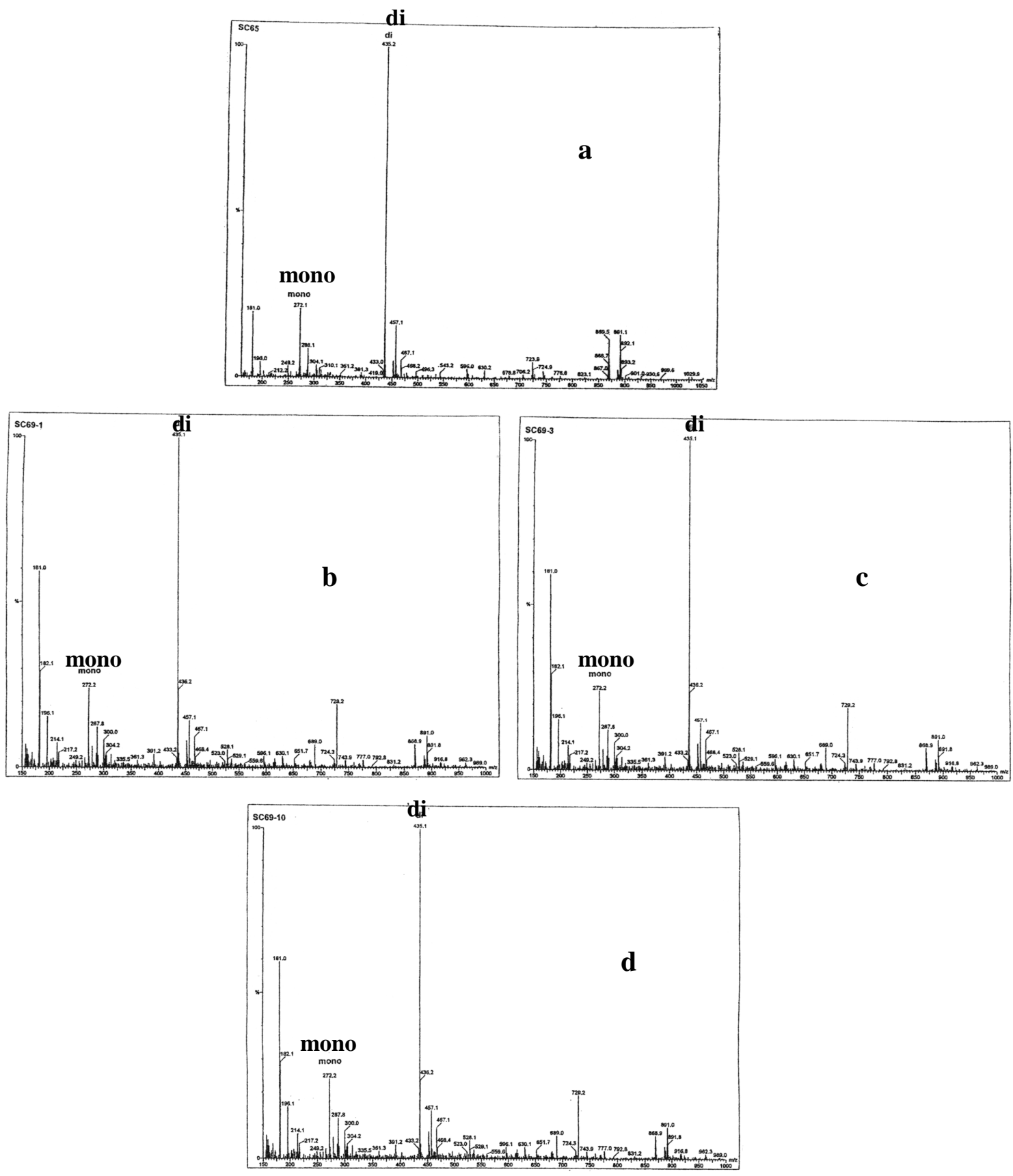

Figure S19. Comparison of the Cl-MS $\mathrm{M}+1$ peaks for the cyclooctene reaction with NHPI in the presence of lead tetraacetate a) original experiment, b) protocol 2, 1 minute prior the addition of NHPI (see page 18), c) protocol 1, 3 minutes prior the addition of NHPI (see page 18), d) protocol 1, 10 minutes prior the addition of NHPI (see page 18) 


\section{X-ray crystallographic data for cyclooctene / PINO mono adduct}

CELL 0.710712 .66738 .121014 .034990 .000106 .42290 .000

$\begin{array}{lllllll}\text { ZERR } 4.00 & 0.0013 & 0.0008 & 0.0014 & 0.000 & 0.002 & 0.000\end{array}$

LATT 1

SYMM 0.5-X, 0.5+Y, 0.5-Z

SFAC C H N O

UNIT 6468412

OMIT 050

BOND

SIZE 0.50 .30 .02

L.S. 8

FMAP 2

PLAN 20

ACTA

TEMP -148

WGHT $\quad 0.048900 \quad 1.679900$

FVAR $\quad 0.493810 .93175$

$\begin{array}{llllllll}\text { O1 } & 4 & 0.176176 & 0.707226 & 0.027824 & 11.00000 & 0.02783 & 0.03251=\end{array}$ $\begin{array}{llll}0.02266 & -0.00034 & -0.00313 & -0.00281\end{array}$

$\begin{array}{llllllll}02 & 4 & -0.033598 & 1.108264 & -0.165107 & 11.00000 & 0.03858 & 0.01832=\end{array}$ $\begin{array}{llll}0.03981 & 0.00247 & 0.01221 & 0.00094\end{array}$

$\begin{array}{llllllll}\mathrm{O} 3 & 4 & 0.145100 & 1.054300 & 0.005190 & 11.00000 & 0.03535 & 0.02750=\end{array}$ $\begin{array}{llll}0.02462 & -0.00985 & 0.01217 & -0.01431\end{array}$

$\begin{array}{llllllll}\mathrm{N} 1 & 3 & 0.089698 & 0.934425 & -0.060202 & 11.00000 & 0.02639 & 0.02214=\end{array}$ $\begin{array}{llll}0.02464 & -0.00467 & 0.00488 & -0.00792\end{array}$

$\begin{array}{llllllll}\mathrm{C} 1 & 1 & 0.104912 & 0.767291 & -0.038145 & 11.00000 & 0.02332 & 0.02369=\end{array}$ $\begin{array}{llll}0.01929 & -0.00137 & 0.00807 & -0.00244\end{array}$

$\begin{array}{llllllll}\mathrm{C} 2 & 1 & 0.014243 & 0.687658 & -0.116415 & 11.00000 & 0.01949 & 0.02197=\end{array}$ $\begin{array}{llll}0.01624 & -0.00065 & 0.00462 & -0.00076\end{array}$

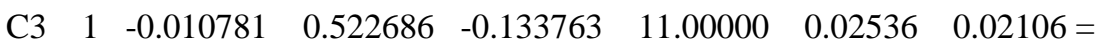
$\begin{array}{llll}0.01966 & 0.00332 & 0.00315 & 0.00201\end{array}$

AFIX 43

$\begin{array}{lllllll}\text { H3 } & 2 & 0.032195 & 0.439674 & -0.092959 & 11.00000 & -1.20000\end{array}$

AFIX 0

$\begin{array}{llllllll}\text { C4 } & 1 & -0.100891 & 0.482652 & -0.212885 & 11.00000 & 0.02725 & 0.02093=\end{array}$ $\begin{array}{llll}0.02510 & -0.00017 & 0.00375 & -0.00244\end{array}$ 
AFIX 43

$\begin{array}{lllllll}\mathrm{H} 4 & 2 & -0.120409 & 0.370248 & -0.225986 & 11.00000 & -1.20000\end{array}$

AFIX 0

$\begin{array}{llllllll}\text { C5 } & 1 & -0.163241 & 0.603957 & -0.273430 & 11.00000 & 0.02454 & 0.02875=\end{array}$ $\begin{array}{llll}0.01963 & -0.00017 & -0.00092 & -0.00147\end{array}$

AFIX 43

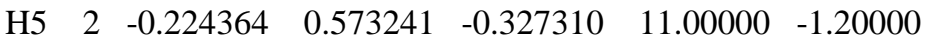

AFIX 0

$\begin{array}{llllllll}\text { C6 } & 1 & -0.137106 & 0.769516 & -0.255968 & 11.00000 & 0.02305 & 0.02247=\end{array}$ $\begin{array}{llll}0.02024 & 0.00390 & 0.00401 & 0.00178\end{array}$

AFIX 43

$\begin{array}{lllllll}\text { H6 } & 2 & -0.178902 & 0.852938 & -0.297331 & 11.00000 & -1.20000\end{array}$

AFIX 0

$\begin{array}{llllllll}\text { C7 } & 1 & -0.048420 & 0.808200 & -0.176526 & 11.00000 & 0.02231 & 0.01963=\end{array}$ $\begin{array}{llll}0.02023 & -0.00003 & 0.00739 & -0.00037\end{array}$

$\begin{array}{llllllll}\text { C8 } & 1 & -0.003432 & 0.972010 & -0.138463 & 11.00000 & 0.02778 & 0.02117=\end{array}$ $\begin{array}{llll}0.02616 & 0.00138 & 0.01495 & 0.00054\end{array}$

EADP C9 C9A

EADP C10 C10A

EADP C11 C11A

EADP C12 C12A

EADP C13 C13A

EADP C14 C14A

EADP C15 C15A

EADP C16 C16A

SADI O3 C9 O3 C9A

DFIX $1.51 \mathrm{C} 9 \mathrm{C} 10 \mathrm{C} 11 \mathrm{C} 12$

DFIX 1.32 C10 C11

DFIX 1.53 C12 C13 C13 C14 C14 C15 C15 C16 C16 C9

DFIX 2.440 .05 C9 C11 C10 C12

DFIX 2.47 0.05 C11 C13 C10 C16

DFIX 2.50 0.05 C12 C14 C14 C16 C13 C15 C15 C9

DFIX 1.51 C9A C10A C11A C12A

DFIX 1.32 C10A C11A

DFIX 1.53 C12A C13A C13A C14A C14A C15A C15A C16A C16A C9A

DFIX 2.44 0.05 C9A C11A C10A C12A 


\section{DFIX 2.47 0.05 C11A C13A C10A C16A}

DFIX 2.50 0.05 C12A C14A C14A C16A C13A C15A C15A C9A

PART 110.93

$\begin{array}{llllllll}\text { C9 } & 1 & 0.223712 & 1.142496 & -0.034468 & 10.93000 & 0.02329 & 0.02382=\end{array}$ $\begin{array}{llll}0.02249 & 0.00092 & 0.00711 & -0.00510\end{array}$

AFIX 13

$\begin{array}{lllllll}\text { H9 } & 2 & 0.183237 & 1.196753 & -0.098305 & 10.93000 & -1.20000\end{array}$

AFIX 0

$\begin{array}{llllllll}\mathrm{C} 10 & 1 & 0.309507 & 1.031005 & -0.052934 & 10.93000 & 0.03142 & 0.03109=\end{array}$ $\begin{array}{llll}0.04947 & -0.00791 & 0.01407 & -0.00573\end{array}$

AFIX 43

$\begin{array}{lllllll}\mathrm{H} 10 & 2 & 0.329317 & 0.934733 & -0.013463 & 10.93000 & -1.20000\end{array}$

AFIX 0

$\begin{array}{llllllll}\text { C11 } & 1 & 0.359039 & 1.061847 & -0.122633 & 10.93000 & 0.05845 & 0.04314=\end{array}$ $\begin{array}{llll}0.07362 & -0.02274 & 0.04296 & -0.01034\end{array}$

AFIX 43

$\begin{array}{lllllll}\mathrm{H} 11 & 2 & 0.416794 & 0.990120 & -0.126847 & 10.93000 & -1.20000\end{array}$

AFIX 0

$\begin{array}{llllllll}\mathrm{C} 12 & 1 & 0.330693 & 1.200990 & -0.194935 & 10.93000 & 0.08120 & 0.05955=\end{array}$ $\begin{array}{llll}0.05027 & -0.01807 & 0.04611 & -0.02530\end{array}$

AFIX 23

$\begin{array}{llllll}\text { H12A } 2 & 0.249672 & 1.213808 & -0.216455 & 10.93000 & -1.20000\end{array}$

$\begin{array}{lllllll}\text { H12B } & 2 & 0.354117 & 1.171349 & -0.254305 & 10.93000 & -1.20000\end{array}$

AFIX 0

$\begin{array}{llllllll}\mathrm{C} 13 & 1 & 0.381992 & 1.366654 & -0.156097 & 10.93000 & 0.06610 & 0.04953=\end{array}$ $\begin{array}{llll}0.05929 & -0.00730 & 0.04186 & -0.01934\end{array}$

AFIX 23

$\begin{array}{llllll}\text { H13A } 2 & 0.461602 & 1.349174 & -0.124679 & 10.93000 & -1.20000\end{array}$

$\begin{array}{lllllll}\text { H13B } & 2 & 0.374853 & 1.440383 & -0.213753 & 10.93000 & -1.20000\end{array}$

AFIX 0

$\begin{array}{llllllll}\text { C14 } & 1 & 0.334918 & 1.455628 & -0.081794 & 10.93000 & 0.05532 & 0.03030=\end{array}$ $\begin{array}{llll}0.05439 & 0.00099 & 0.02847 & -0.00803\end{array}$

AFIX 23

$\begin{array}{llllll}\text { H14A } 2 & 0.253959 & 1.461407 & -0.109661 & 10.93000 & -1.20000\end{array}$

$\begin{array}{llllll}\text { H14B } 2 & 0.363123 & 1.569997 & -0.074661 & 10.93000 & -1.20000\end{array}$

AFIX 0

$\begin{array}{llllllll}\mathrm{C} 15 & 1 & 0.360907 & 1.378033 & 0.022059 & 10.93000 & 0.04118 & 0.03471=\end{array}$ $\begin{array}{llll}0.03968 & -0.00900 & 0.01378 & -0.01402\end{array}$ 
AFIX 23

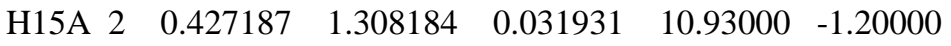

$\begin{array}{lllllll}\text { H15B } & 2 & 0.379498 & 1.467547 & 0.071902 & 10.93000 & -1.20000\end{array}$

AFIX 0

$\begin{array}{llllllll}\mathrm{C} 16 & 1 & 0.270137 & 1.274350 & 0.042776 & 10.93000 & 0.02584 & 0.02717=\end{array}$ $\begin{array}{llll}0.02071 & -0.00010 & 0.00069 & -0.00464\end{array}$

AFIX 23

$\begin{array}{llllll}\text { H16A } 2 & 0.209429 & 1.348130 & 0.047057 & 10.93000 & -1.20000\end{array}$

$\begin{array}{lllllll}\text { H16B } & 2 & 0.299218 & 1.220595 & 0.108286 & 10.93000 & -1.20000\end{array}$

AFIX 0

PART 210.07

$\begin{array}{llllllll}\text { C9A } & 1 & 0.227805 & 1.127998 & -0.036268 & 10.07000 & 0.02329 & 0.02382=\end{array}$ $\begin{array}{llll}0.02249 & 0.00092 & 0.00711 & -0.00510\end{array}$

AFIX 13

$\begin{array}{lllllll}\text { H9A } & 2 & 0.296025 & 1.059142 & -0.019960 & 10.07000 & -1.20000\end{array}$

AFIX 0

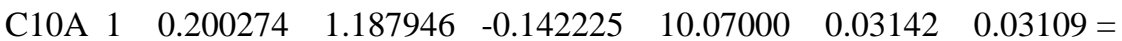
$\begin{array}{llll}0.04947 & -0.00791 & 0.01407 & -0.00573\end{array}$

AFIX 43

$\begin{array}{lllllll}\text { H10A } 2 & 0.127400 & 1.222914 & -0.174675 & 10.07000 & -1.20000\end{array}$

AFIX 0

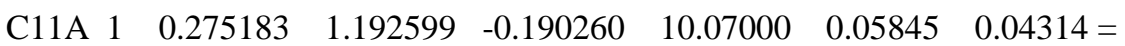
$\begin{array}{llll}0.07362 & -0.02274 & 0.04296 & -0.01034\end{array}$

AFIX 43

$\begin{array}{lllllll}\text { H11A } & 2 & 0.255852 & 1.236541 & -0.255651 & 10.07000 & -1.20000\end{array}$

AFIX 0

$\begin{array}{llllllll}\text { C12A } & 1 & 0.390399 & 1.131073 & -0.145794 & 10.07000 & 0.08120 & 0.05955=\end{array}$ $\begin{array}{llll}0.05027 & -0.01807 & 0.04611 & -0.02530\end{array}$

AFIX 23

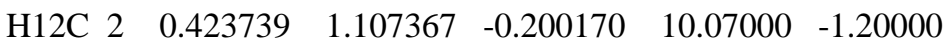

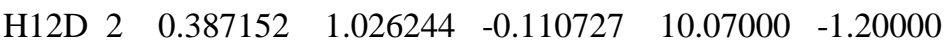

AFIX 0

$\begin{array}{llllllll}\text { C13A } & 1 & 0.464301 & 1.249930 & -0.073738 & 10.07000 & 0.06610 & 0.04953=\end{array}$ $\begin{array}{llll}0.05929 & -0.00730 & 0.04186 & -0.01934\end{array}$

AFIX 23

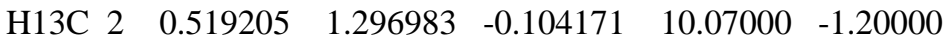

$\begin{array}{llllll}\text { H13D } 2 & 0.504405 & 1.190283 & -0.012721 & 10.07000 & -1.20000\end{array}$

AFIX 0 


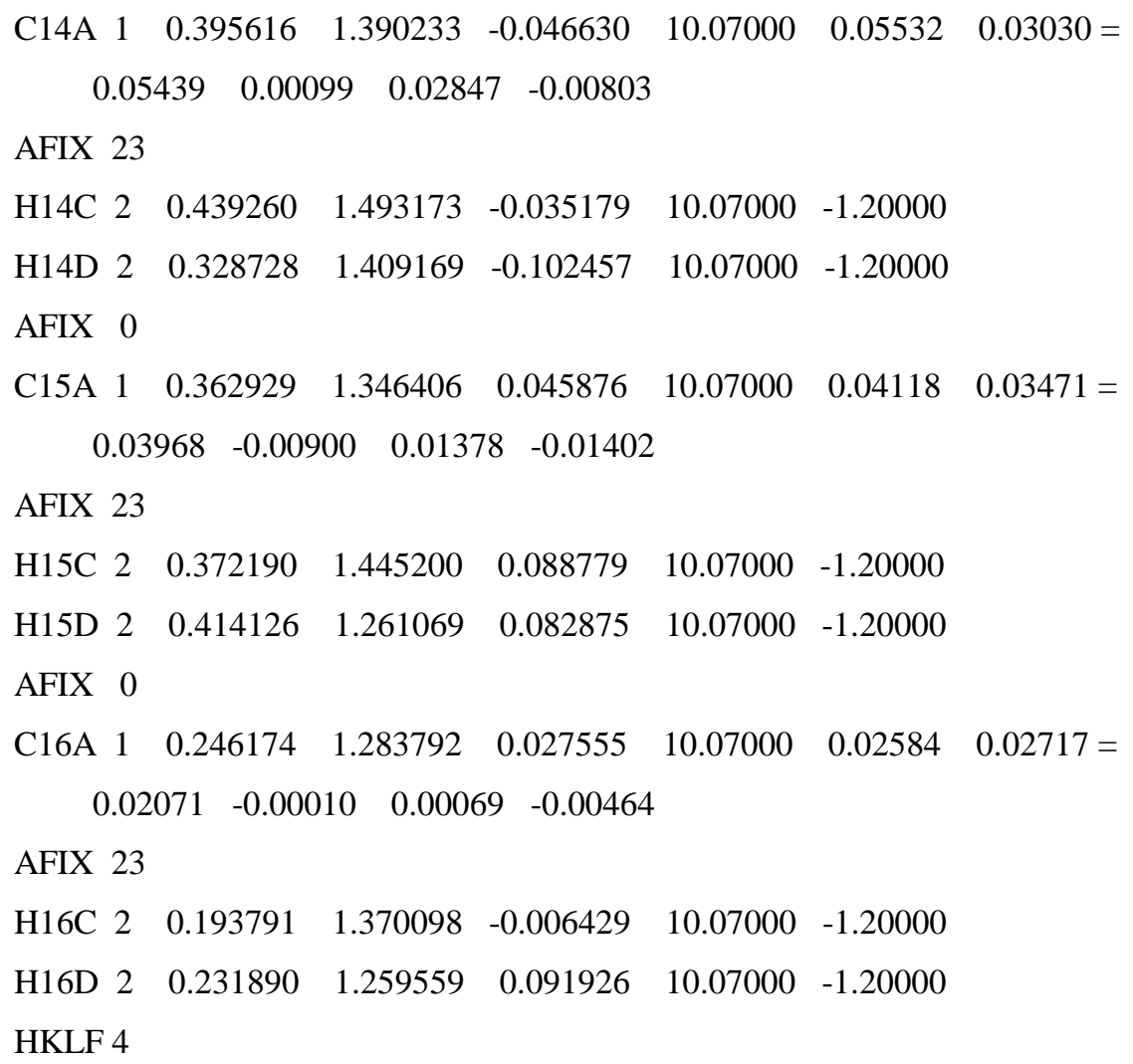


$\begin{array}{lllllllll}\text { Q12 } & 1 & 0.2344 & 0.7591 & 0.0925 & 11.00000 & 0.05 & 0.18 \\ \text { Q13 } & 1 & -0.1213 & 0.6988 & -0.2856 & 11.00000 & 0.05 & 0.17 \\ \text { Q14 } & 1 & 0.0216 & 0.6237 & -0.1442 & 11.00000 & 0.05 & 0.17 \\ \text { Q15 } & 1 & 0.0874 & 0.3913 & -0.0595 & 11.00000 & 0.05 & 0.17 \\ \text { Q16 } & 1 & 0.2816 & 1.3743 & 0.0145 & 11.00000 & 0.05 & 0.17 \\ \text { Q17 } & 1 & 0.2575 & 1.2037 & 0.0070 & 11.00000 & 0.05 & 0.17 \\ \text { Q18 } & 1 & 0.4486 & 0.9487 & -0.1018 & 11.00000 & 0.05 & 0.16 \\ \text { Q19 } & 1 & 0.4871 & 1.1891 & -0.0532 & 11.00000 & 0.05 & 0.16 \\ \text { Q20 } & 1 & 0.2061 & 1.3854 & 0.0707 & 11.00000 & 0.05 & 0.16\end{array}$ 


\section{X-ray crystallographic data for cyclooctene / PINO di-adduct}

CELL 0.710710 .70235 .715317 .046690 .000102 .98290 .000

$\begin{array}{lllllll}\text { ZERR } 2.00 & 0.0004 & 0.0002 & 0.0006 & 0.000 & 0.001 & 0.000\end{array}$

LATT -1

SYMM 0.5+X, $-\mathrm{Y}, 0.5+\mathrm{Z}$

SFAC C H N O

UNIT 4844412

BOND

L.S. 8

FMAP 2

PLAN 20

SIZE 0.50 .40 .06

ACTA

TEMP -148

WGHT $\quad 0.050200 \quad 0.136500$

FVAR $\quad 0.84221$

$\begin{array}{llllllll}\text { O1 } & 4 & 0.274136 & -0.019324 & 0.179337 & 11.00000 & 0.02863 & 0.03787=\end{array}$ $\begin{array}{llll}0.02577 & -0.00653 & 0.01126 & -0.00852\end{array}$

$\begin{array}{llllllll}\mathrm{O} 2 & 4 & 0.053573 & 0.096417 & 0.377017 & 11.00000 & 0.02518 & 0.02978=\end{array}$ $\begin{array}{llll}0.02462 & -0.00274 & 0.00834 & 0.00228\end{array}$

$\begin{array}{llllllll}\text { O3 } & 4 & 0.265792 & 0.257055 & 0.314048 & 11.00000 & 0.01918 & 0.01902=\end{array}$ $\begin{array}{llll}0.02543 & -0.00348 & 0.00083 & -0.00077\end{array}$

$\begin{array}{llllllll}\mathrm{O} 4 & 4 & 0.241129 & 0.661200 & 0.424298 & 11.00000 & 0.02877 & 0.02018=\end{array}$ $\begin{array}{llll}0.02880 & 0.00201 & 0.01005 & -0.00189\end{array}$

$\begin{array}{llllllll}05 & 4 & 0.294676 & 0.021271 & 0.590327 & 11.00000 & 0.03566 & 0.02794=\end{array}$ $\begin{array}{llll}0.02903 & 0.00729 & 0.00765 & 0.00865\end{array}$

$\begin{array}{llllllll}06 & 4 & 0.411614 & 0.296555 & 0.486656 & 11.00000 & 0.01717 & 0.02690=\end{array}$ $\begin{array}{llll}0.02476 & -0.00615 & 0.00552 & -0.00013\end{array}$

$\begin{array}{llllllll}\mathrm{N} 1 & 3 & 0.191457 & 0.061635 & 0.290161 & 11.00000 & 0.01990 & 0.02306=\end{array}$ $\begin{array}{llll}0.02118 & -0.00313 & 0.00477 & -0.00246\end{array}$

$\begin{array}{llllllll}\mathrm{N} 2 & 3 & 0.290587 & 0.325371 & 0.499966 & 11.00000 & 0.01984 & 0.02395=\end{array}$ $\begin{array}{llll}0.02207 & 0.00168 & 0.00586 & 0.00330\end{array}$

$\begin{array}{llllllll}\text { C1 } & 1 & 0.198626 & -0.060019 & 0.220430 & 11.00000 & 0.02168 & 0.02394=\end{array}$ $\begin{array}{llll}0.01945 & -0.00309 & 0.00185 & -0.00145\end{array}$

$\begin{array}{llllllll}\text { C2 } & 1 & 0.096519 & -0.240592 & 0.212197 & 11.00000 & 0.01841 & 0.02452=\end{array}$ 


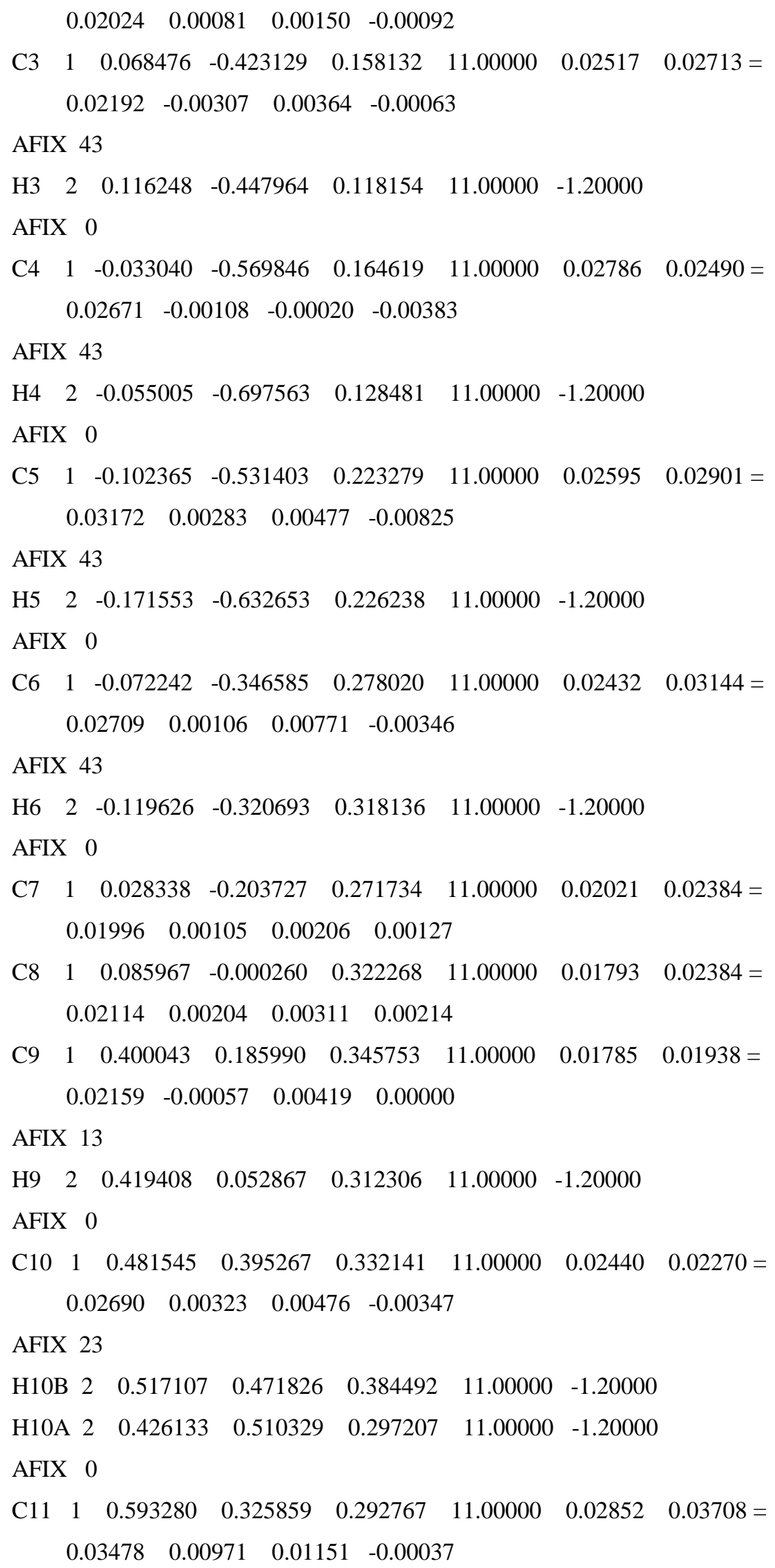


AFIX 23

$\begin{array}{llllll}\text { H11B } 2 & 0.557982 & 0.305954 & 0.234292 & 11.00000 & -1.20000\end{array}$

$\begin{array}{lllllll}\text { H11A } 2 & 0.654370 & 0.458318 & 0.299437 & 11.00000 & -1.20000\end{array}$

AFIX 0

$\begin{array}{llllllll}\mathrm{C} 12 & 1 & 0.668513 & 0.105240 & 0.324027 & 11.00000 & 0.02809 & 0.03679=\end{array}$ $\begin{array}{llll}0.02893 & 0.00121 & 0.01218 & 0.00432\end{array}$

AFIX 23

$\begin{array}{lllllll}\text { H12B } 2 & 0.607467 & -0.027226 & 0.317778 & 11.00000 & -1.20000\end{array}$

$\begin{array}{lllllll}\mathrm{H} 12 \mathrm{~A} & 2 & 0.729079 & 0.072772 & 0.289194 & 11.00000 & -1.20000\end{array}$

AFIX 0

$\begin{array}{llllllll}\mathrm{C} 13 & 1 & 0.744258 & 0.109375 & 0.411107 & 11.00000 & 0.02216 & 0.04718=\end{array}$ $\begin{array}{llll}0.03251 & 0.00290 & 0.00677 & 0.00671\end{array}$

AFIX 23

$\begin{array}{llllll}\text { H13B } 2 & 0.808101 & 0.237155 & 0.416662 & 11.00000 & -1.20000\end{array}$

$\begin{array}{lllllll}\text { H13A } 2 & 0.792409 & -0.039417 & 0.421954 & 11.00000 & -1.20000\end{array}$

AFIX 0

$\begin{array}{llllllll}\mathrm{C} 14 & 1 & 0.668176 & 0.142043 & 0.476157 & 11.00000 & 0.02225 & 0.03707=\end{array}$ $\begin{array}{llll}0.02490 & -0.00010 & 0.00285 & 0.00448\end{array}$

AFIX 23

H14B $2 \quad 0.641007 \quad 0.307692 \quad 0.475804 \quad 11.00000 \quad-1.20000$

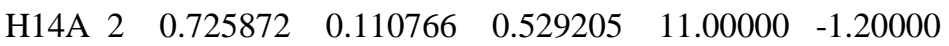

AFIX 0

$\begin{array}{llllllll}\mathrm{C} 15 & 1 & 0.547866 & -0.014835 & 0.467603 & 11.00000 & 0.02669 & 0.02495=\end{array}$ $\begin{array}{llll}0.02465 & 0.00411 & 0.00638 & 0.00612\end{array}$

AFIX 23

$\begin{array}{llllll}\text { H15B } 2 & 0.557723 & -0.150348 & 0.433326 & 11.00000 & -1.20000\end{array}$

$\begin{array}{lllllll}\text { H15A } 2 & 0.545590 & -0.076456 & 0.521542 & 11.00000 & -1.20000\end{array}$

AFIX 0

$\begin{array}{llllllll}\text { C16 } & 1 & 0.418535 & 0.100966 & 0.432136 & 11.00000 & 0.02038 & 0.01906=\end{array}$ $\begin{array}{llll}0.02064 & -0.00022 & 0.00481 & -0.00055\end{array}$

AFIX 13

$\begin{array}{lllllll}H 16 & 2 & 0.348682 & -0.012710 & 0.435031 & 11.00000 & -1.20000\end{array}$

AFIX 0

$\begin{array}{llllllll}\mathrm{C} 17 & 1 & 0.216960 & 0.522558 & 0.471476 & 11.00000 & 0.01946 & 0.02021=\end{array}$ $\begin{array}{llll}0.02185 & -0.00353 & 0.00351 & -0.00157\end{array}$

$\begin{array}{llllllll}\mathrm{C} 18 & 1 & 0.109216 & 0.513070 & 0.513767 & 11.00000 & 0.02109 & 0.02188=\end{array}$ $\begin{array}{llll}0.02034 & -0.00197 & 0.00539 & -0.00205\end{array}$

$\begin{array}{llllllll}\mathrm{C} 19 & 1 & 0.009919 & 0.668894 & 0.510294 & 11.00000 & 0.02482 & 0.02439=\end{array}$ 
$\begin{array}{llll}0.02662 & 0.00012 & 0.00584 & 0.00208\end{array}$

AFIX 43

$\begin{array}{lllllll}\mathrm{H} 19 & 2 & 0.001192 & 0.804484 & 0.477272 & 11.00000 & -1.20000\end{array}$

AFIX 0

$\begin{array}{llllllll}\mathrm{C} 20 & 1 & -0.077699 & 0.617313 & 0.557979 & 11.00000 & 0.02273 & 0.03288=\end{array}$ $\begin{array}{llll}0.02933 & -0.00436 & 0.00712 & 0.00292\end{array}$

AFIX 43

$\begin{array}{lllllll}\mathrm{H} 20 & 2 & -0.147326 & 0.720713 & 0.557618 & 11.00000 & -1.20000\end{array}$

AFIX 0

$\begin{array}{llllllll}\mathrm{C} 21 & 1 & -0.064500 & 0.417971 & 0.605667 & 11.00000 & 0.02553 & 0.03541=\end{array}$ $\begin{array}{llll}0.02538 & -0.00382 & 0.01031 & -0.00361\end{array}$

AFIX 43

$\begin{array}{lllllll}\mathrm{H} 21 & 2 & -0.125838 & 0.386848 & 0.636875 & 11.00000 & -1.20000\end{array}$

AFIX 0

$\begin{array}{llllllll}\mathrm{C} 22 & 1 & 0.036883 & 0.262443 & 0.608734 & 11.00000 & 0.02957 & 0.02632=\end{array}$ $\begin{array}{llll}0.02290 & 0.00007 & 0.00843 & -0.00356\end{array}$

AFIX 43

$\begin{array}{lllllll}\mathrm{H} 22 & 2 & 0.045615 & 0.125530 & 0.641112 & 11.00000 & -1.20000\end{array}$

AFIX 0

$\begin{array}{llllllll}\mathrm{C} 23 & 1 & 0.123955 & 0.315733 & 0.562783 & 11.00000 & 0.02264 & 0.02189=\end{array}$ $\begin{array}{llll}0.02013 & -0.00114 & 0.00400 & 0.00156\end{array}$

$\begin{array}{llllllll}\mathrm{C} 24 & 1 & 0.244400 & 0.192625 & 0.556477 & 11.00000 & 0.02416 & 0.02321=\end{array}$ $\begin{array}{llll}0.01990 & -0.00148 & 0.00508 & 0.00057\end{array}$

HKLF 4

REM Serg2 in Pn

REM R1 $=0.0295$ for $4752 \mathrm{Fo}>4 \operatorname{sig}(\mathrm{Fo})$ and 0.0304 for all 4887 data

REM 289 parameters refined using 2 restraints

END

WGHT $\quad 0.0505 \quad 0.1349$

REM Highest difference peak 0.233 , deepest hole -0.218, 1-sigma level 0.035

$\begin{array}{llllllll}\text { Q1 } & 1 & 0.6355 & -0.0741 & 0.4084 & 11.00000 & 0.05 & 0.23\end{array}$

$\begin{array}{llllllll}\text { Q2 } & 1 & 0.4420 & 0.2906 & 0.3406 & 11.00000 & 0.05 & 0.23\end{array}$

$\begin{array}{llllllll}\text { Q3 } & 1 & 0.1610 & 0.5274 & 0.4934 & 11.00000 & 0.05 & 0.22\end{array}$

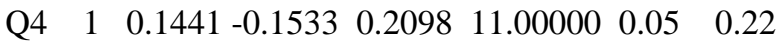

$\begin{array}{llllllllll}\text { Q5 } & 1 & 0.5876 & 0.4173 & 0.3302 & 11.00000 & 0.05 & 0.22\end{array}$ 


$\begin{array}{llllllll}\text { Q6 } & 1 & 0.1752 & 0.2446 & 0.5597 & 11.00000 & 0.05 & 0.21 \\ \text { Q7 } & 1 & 0.4117 & 0.1483 & 0.3918 & 11.00000 & 0.05 & 0.20 \\ \text { Q8 } & 1 & 0.0818 & -0.2581 & 0.2508 & 11.00000 & 0.05 & 0.19 \\ \text { Q9 } & 1 & 0.0628 & -0.2161 & 0.2416 & 11.00000 & 0.05 & 0.19 \\ \text { Q10 } & 1 & 0.6816 & 0.3838 & 0.4109 & 11.00000 & 0.05 & 0.18 \\ \text { Q11 } & 1 & 0.1018 & 0.3846 & 0.5243 & 11.00000 & 0.05 & 0.17 \\ \text { Q12 } & 1 & 0.2657 & 0.4299 & 0.4901 & 11.00000 & 0.05 & 0.17 \\ \text { Q13 } & 1 & -0.0622 & -0.5527 & 0.1992 & 11.00000 & 0.05 & 0.17 \\ \text { Q14 } & 1 & 0.7034 & 0.1295 & 0.4403 & 11.00000 & 0.05 & 0.17 \\ \text { Q15 } & 1 & 0.4846 & 0.0435 & 0.4467 & 11.00000 & 0.05 & 0.17 \\ \text { Q16 } & 1 & 0.2869 & 0.2908 & 0.5372 & 11.00000 & 0.05 & 0.16 \\ \text { Q17 } & 1 & -0.0262 & 0.6478 & 0.5443 & 11.00000 & 0.05 & 0.16 \\ \text { Q18 } & 1 & -0.0659 & -0.4754 & 0.2608 & 11.00000 & 0.05 & 0.16 \\ \text { Q19 } & 1 & 0.0218 & -0.5035 & 0.1697 & 11.00000 & 0.05 & 0.16 \\ \text { Q20 } & 1 & 0.7020 & 0.1182 & 0.3706 & 11.00000 & 0.05 & 0 .\end{array}$

